\title{
Compressible Turbulence Transport Equations for Generalized Second Order Closure
}

\author{
Lawrence D. Cloutman
}

May 1999

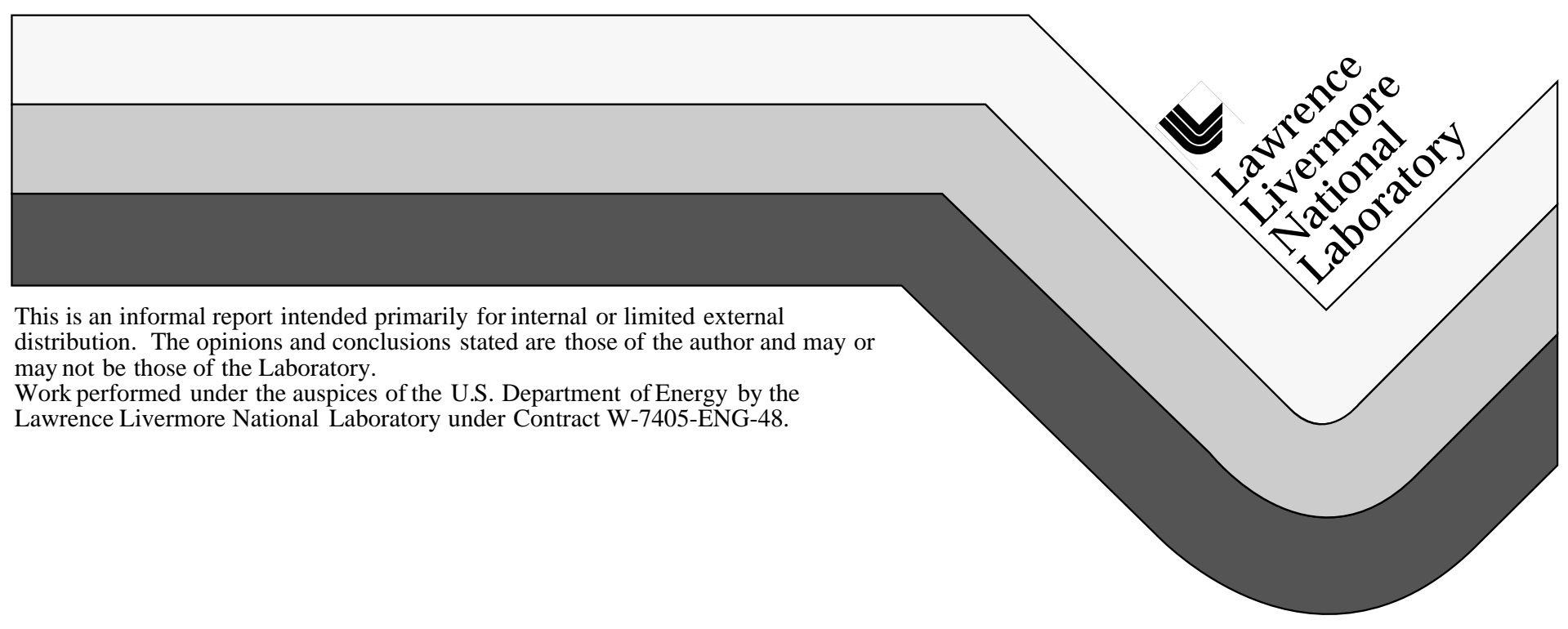




\section{DISCLAIMER}

This document was prepared as an account of work sponsored by an agency of the United States Government. Neither the United States Government nor the University of California nor any of their employees, makes any warranty, express or implied, or assumes any legal liability or responsibility for the accuracy, completeness, or usefulness of any information, apparatus, product, or process disclosed, or represents that its use would not infringe privately owned rights. Reference herein to any specific commercial product, process, or service by trade name, trademark, manufacturer, or otherwise, does not necessarily constitute or imply its endorsement, recommendation, or favoring by the United States Government or the University of California. The views and opinions of authors expressed herein do not necessarily state or reflect those of the United States Government or the University of California, and shall not be used for advertising or product endorsement purposes.

This report has been reproduced directly from the best available copy.

Available to DOE and DOE contractors from the Office of Scientific and Technical Information P.O. Box 62, Oak Ridge, TN 37831

Prices available from (423) 576-8401

Available to the public from the National Technical Information Service

U.S. Department of Commerce 5285 Port Royal Rd., Springfield, VA 22161 
Lawrence Livermore National Laboratory Report

UCRL-ID-134075

May 1999

\title{
COMPRESSIBLE TURBULENCE TRANSPORT EQUATIONS FOR GENERALIZED SECOND ORDER CLOSURE
}

\author{
Lawrence D. Cloutman \\ Lawrence Livermore National Laboratory \\ Livermore, California 94550
}

\begin{abstract}
Progress on the theory of second order closure in turbulence models of various types requires knowledge of the transport equations for various turbulence correlations. This report documents a procedure that provides such equations for a wide variety of turbulence averages for compressible flows of a multicomponent fluid. Generalizing some work by Germano for incompressible flows, we introduce an appropriate extension of his generalized second order correlations and use a generalized mass-weighted averaging procedure to derive transport equations for the correlations. The averaging procedure includes all of the commonly used averages as special cases. The resulting equations provide an internally consistent starting point for future work in developing single-point statistical turbulence transport models for fluid flows. The form invariance of the incompressible equations also holds for the compressible case, and we discuss some of the closure issues and frequently ignored complications of statistical turbulence models of compressible flows.
\end{abstract}




\section{Introduction}

Turbulence is perhaps the most important unsolved problem in fluid dynamics. There are large uncertainties in the theoretical rates of heat transfer, turbulent mixing, and other phenomena of practical interest. Unfortunately, progress in understanding turbulence has been agonizingly slow. The situation is described succinctly by Cebeci and Smith [1], p. ix: "Fluid mechanics is beset by the difficulty that man's ability to write the governing equations of motion far outruns his ability to solve them. This difficulty is an especially annoying handicap in the case of turbulent flows." Ideally, we would like to solve the Navier-Stokes equations with sufficient resolution to predict the behavior of even the smallest flow features at the Kolmogorov microscale. This approach is called "direct numerical simulation" (DNS), and it requires three-dimensional Navier-Stokes calculations with at least $\operatorname{Re}^{0.75}$ zones in each direction, where Re is the Reynolds number. ${ }^{1}$ In practice, with Re taking values from approximately 2000 for transitional flows up to $10^{9}$ or more in stellar convection zones, present computational resources are inadequate for a true DNS in most cases. This situation is exacerbated by the need to use real-gas constitutive relations in many applications.

To make the problem tractable, we introduce simplifying techniques and approximations generically known as "turbulence models." The models that are practical from engineering and computational standpoints usually are based on some kind of averaging or convolution of the flow field to suppress the small-scale features. This procedure leads to averaged governing partial differential equations containing a number of unknown functionals of the flow field. The challenge for turbulence modelers is to close the system of equations by finding accurate approximations for these unknown functionals. The main point of this report is that one may derive transport equations for some of these functionals by manipulation of the original equations, and that this process results in transport equations that have the same form regardless of the type of averaging, provided the functionals are defined in a particular way. This is accomplished by generalizing the results of Germano [2] to compressible multicomponent flows.

We adopt the working hypothesis that the Navier-Stokes equations for multicomponent flows listed in Section 3 describe all of the details of turbulent flows. Therefore in one sense, there is no such thing as turbulence, just very complicated laminar flows that fall in the category of deterministic chaos. This viewpoint implies that we are free to define turbulence in any manner we find convenient or useful. What we are trying to accomplish

\footnotetext{
${ }^{1}$ There are those who apply the term DNS to numerical simulations that neither resolve all scales nor use a turbulence model. A colleague who shall rename anonymous has dubbed this approach a BSDNS, quite justifiably in my opinion. A variation on this approach is to use a filter or smoothing operation on the smallest resolved scales. However, this is precisely what we try to accomplish with a large eddy simulation. We limit the use of the label DNS to refer to a fully resolved Navier-Stokes numerical simulation.
} 
Table 1.

Turbulence Averaging Operators

Type of Average: Definition

Time Average : $\tilde{\rho}(\mathbf{r}, t)=\lim _{\tau \rightarrow \infty} \frac{1}{2 \tau} \int_{t-\tau}^{t+\tau} \rho(\mathbf{r}, \theta) d \theta$

Ensemble Average : $\tilde{\rho}(\mathbf{r}, t)=\lim _{N \rightarrow \infty} \frac{1}{N} \sum_{\omega=1}^{N} \rho_{\omega}(\mathbf{r}, t)$

Horizontal (Spherical) Average : $\tilde{\rho}(r, t)=\frac{1}{4 \pi} \int_{0}^{2 \pi} \int_{0}^{\pi} \rho(\mathbf{r}, t) \sin \theta d \theta d \phi$

Schumann/SGS: $\tilde{\rho}_{i, j, k}(t)=\int_{-\delta x_{i} / 2}^{\delta x_{i} / 2} \int_{-\delta y_{j} / 2}^{\delta y_{j} / 2} \int_{-\delta z_{k} / 2}^{\delta z_{k} / 2} \rho\left(\mathbf{r}^{\prime}, t\right) d x^{\prime} d y^{\prime} d z^{\prime}$

LES/SGS : $\tilde{\rho}(\mathbf{r}, t)=\int_{-\infty}^{\infty} \int_{-\infty}^{\infty} \int_{-\infty}^{\infty} \rho\left(\mathbf{r}^{\prime}, t\right) G\left(\mathbf{r}-\mathbf{r}^{\prime} ; L\right) d x^{\prime} d y^{\prime} d z^{\prime}$

e.g., $\quad G\left(\mathbf{r}-\mathbf{r}^{\prime} ; L\right)=\frac{1}{\pi^{3 / 2} L^{3}} \exp \left(-\left|\mathbf{r}-\mathbf{r}^{\prime}\right|^{2} / L^{2}\right)$

with a turbulence model is to suppress enough of the detailed small-scale information to make the computational problem tractable. Usually this is done by introducing an averaging operator to split the total flow field into a mean flow (which should be sufficiently smooth that we can simulate it with a computational fluid dynamics calculation) and a turbulent fluctuation (which we parameterize or approximate in some fashion with a turbulence model). The choice of averaging operator is not unique, and Table 1 lists several commonly used operators. Once an appropriate averaging operator is selected, the governing equations are averaged to produce the mean flow equations. These equations contain correlations between various turbulent fluctuations, and additional equations must be introduced to allow estimation of the values of these correlations. These additional equations are usually obtained either by appropriate manipulation of the orginal governing equations or by hypothesizing algebraic relations among turbulent correlations and various functions of the mean flow. These additional equations make up the turbulence model, which must be carefully validated by comparing its predictions with a wide variety of experimental data. Good introductory descriptions of this approach are given by Reynolds [3, 4] and by Launder and Spalding [5, 6]. Jones [7] gives a more detailed discussion of ensemble averaging.

Turbulence defined as the flow features eliminated by an averaging operation has the 
interesting characteristic that it appears to be stochastic when examined in detail, even at small wavenumbers. This physical characteristic is mirrored in the mathematical nature of the governing partial differential equations: although deterministic, they exhibit chaotic solutions for sufficiently high values of a characteristic dimensionless parameter such as the Reynolds number or Rayleigh number. This is why turbulent flows have limited predictability. The solutions are sensitive to small perturbations in the initial or boundary conditions, and two realizations of an experiment started with infinitesimal differences in their initial conditions will diverge after a finite time. That is, details of these flows can be predicted only for a limited time into the future, no matter how accurate the initial data. This difficulty applies both to numerical and theoretical studies, where there are at least three sources of uncontrolled perturbations. First, we do not have the capability of producing exact solutions of the transient, nonlinear governing equations. Truncation and rounding errors are found in all numerical solutions. Second, the governing fluid flow equations themselves are not exact. For example, we usually assume that relativistic and quantum mechanical effects enter only through the constitutive relations. We also assume the fluid is a continuum, while in reality it is a collection of a finite number of atoms and molecules that are subject to tiny "random" statistical fluctuations. Third, we can never know the constitutive relations, initial conditions, and boundary conditions with infinite precision. This extreme sensitivity explains why, for example, weather forecasts are so unreliable and have dismal prospects for ever being reliable for periods beyond a few days. Thus we are forced to abandon strictly deterministic prediction in favor of attempting to predict only the large-scale (possibly coherent) structures in the flows and certain statistical quantities (that is, functionals of the fluctuating, stochastic turbulent components). Even in a DNS, the predictability problem interferes with detailed comparisons between experiments and simulations. Even the integral scale may be affected (as in weather prediction).

In recent years considerable progress has been made in the development of turbulence models for engineering purposes. The most commonly used models employ from zero to two transport equations for selected turbulence variables, and algebraic closure relations are used for the remaining unknown correlations. One of these models, the $k-\epsilon$ model [8], has been widely used in engineering studies of a variety of turbulent flows. Different versions of this model are based on different averages, with time and ensemble averages being the most common. These models employ transport equations for the turbulence kinetic energy density $k$ and a turbulence decay rate function $\epsilon$. Variations of this basic model transport variables other than $\epsilon$, such as a length scale or a vorticity density. Large eddy simulation (LES) models also have been used with good success, and these are based on the filter function approach. Most LES models use an algebraic closure, although a few employ a 
turbulence kinetic energy transport equation. In all models, there are turbulent correlations that must be approximated algebraically. The goal of this report is to present generalized transport equations for the important second order correlations for a broad class of averages that include $k-\epsilon$ and LES models as special cases. This is a first step in developing new and improved models, either by providing closed transport equations or by providing physical insight to aid the development of algebraic closures.

In Section 2, we demonstrate the generalized averaging process using some model equations. Section 3 presents the multicomponent Navier-Stokes equations and some typical constitutive relations. In Section 4, we apply the results of Section 2 to the Navier-Stokes equations to derive the transport equations required in second order closure models. These equations are the fundamental starting point for all the common single-point statistical turbulence transport models for fluid flows. Section 5 comments on selected closure approximations, and Section 6 contains some closing remarks. There are several appendices that supply additional details about selected topics in the main text.

\section{Model Transport Equations}

This section presents a generalization to compressible multicomponent flows of the ideas of Germano [2]. The basic governing equations of fluid dynamics are the Navier-Stokes equations, and they can be written in the following generic forms:

$$
\frac{\partial \rho}{\partial t}+\nabla \cdot(\rho \mathbf{u})=0
$$

and

$$
\frac{\partial \rho f}{\partial t}+\nabla \cdot(\rho f \mathbf{u})=S_{f}
$$

where $\rho$ is the density, $t$ is time, $f$ is a scalar function, $\mathbf{u}$ is the velocity, and $S_{f}$ is the diffusion and source terms. It is easy to show (with the aid of the vector relations listed in Appendix A) that for two scalar functions, $f$ and $g$, each a solution of equation 2 , the product $f g$ is a solution of the equation

$$
\frac{\partial \rho f g}{\partial t}+\nabla \cdot(\rho f g \mathbf{u})=g S_{f}+f S_{g}
$$

We can do the same thing when $f$ is a vector function. In practice, $\mathbf{u}$ is the only vector function of interest, and

$$
\frac{\partial \rho \mathbf{u}}{\partial t}+\nabla \cdot(\rho \mathbf{u u})=\mathbf{S}_{\mathbf{u}}
$$

The transport equation for the product of a scalar $f$ with $\mathbf{u}$ is

$$
\frac{\partial \rho f \mathbf{u}}{\partial t}+\nabla \cdot(\rho f \mathbf{u u})=f \mathbf{S}_{\mathbf{u}}+S_{f} \mathbf{u}
$$


There are two products of $\mathbf{u}$ of interest, the dyadic $\mathbf{u u}$ and the dot product $\mathbf{u} \cdot \mathbf{u}$. The transport equations are

$$
\frac{\partial \rho \mathbf{u u}}{\partial t}+\nabla \cdot[\rho \mathbf{u}(\mathbf{u u})]=\mathbf{u} \mathbf{S}_{\mathbf{u}}+\mathbf{S}_{\mathbf{u}} \mathbf{u}
$$

and

$$
\left.\frac{\partial \rho \mathbf{u} \cdot \mathbf{u}}{\partial t}+\nabla \cdot[\rho(\mathbf{u} \cdot \mathbf{u}) \mathbf{u})\right]=2 \mathbf{u} \cdot \mathbf{S}_{\mathbf{u}}
$$

Equation 7 is simply the contraction of equation 6 .

The commonly used averaging operations in turbulence modeling can be written in the single general form

$$
\langle f(\mathbf{r}, t)\rangle=\iiint \iint f_{\omega}\left(\mathbf{r}^{\prime}, t^{\prime}\right) G\left(\mathbf{r}^{\prime}-\mathbf{r}, t^{\prime}-t ; L, \Theta ; \omega\right) d^{3} \mathbf{r}^{\prime} d t^{\prime} d \omega
$$

where $f_{\omega}$ is $f$ for the $\omega$ th realization and $G$ is the averaging kernel with an associated length scale $L$ and time scale $\Theta$. Time averages, spatial averages, and large eddy simulation (LES) filtering are included in this general form by appropriate choices of the kernel $G$ and the integration limits. The parameter $\omega$ allows us to include ensemble and cycle averages (sometimes used for periodic systems such as internal combustion engines) in this formalism. It is basically a cycle or realization counter taken to the limit of an infinite number of realizations. For time or space averages, the $\omega$ dependence of the kernel is $\delta(1-\omega)$, and the $\omega=1$ (that is, the first) realization is all that is included in the average.

Regardless of the type of filter used, the filtering process must be accomplished by a linear operator that commutes with space and time derivatives. It is obvious that this condition is met for all the averages shown in Table 1 except for the two sub-grid scale (SGS) models. Appendix B discusses the conditions under which the filter function operator commutes with space and time derivatives. The Schumann approach [9] is different. This model defines averaged quantities only on a finite set of grid points, and the commutation issue never arises.

We use a mass-weighted average. ${ }^{2}$ Beginning with the density,

$$
\langle\rho(\mathbf{r}, t)\rangle \equiv \tilde{\rho}(\mathbf{r}, t) .
$$

The turbulent component $\rho^{\prime}(\mathbf{r}, t) \equiv \rho-\tilde{\rho}$ has a zero average value. If $f$ is any variable except $\rho$ or the pressure $P$, the average is mass-weighted:

$$
\langle\rho(\mathbf{r}, t) f(\mathbf{r}, t)\rangle=\tilde{\rho}(\mathbf{r}, t) \bar{f}(\mathbf{r}, t) .
$$

Germano notes that we would prefer to have averaging operations that obey the relations

$$
\langle\rho f \bar{g}\rangle=\tilde{\rho} \bar{f} \bar{g}
$$

\footnotetext{
${ }^{2}$ Mass-weighted averages are often called Favre averages [10, 11]. However, Cowling [12] clearly distinguished between mass-weighted and non-mass-weighted averages at a much earlier date .
} 
Table 2.

Mass Weighted Averages of Products of Variables

\begin{tabular}{ll}
\hline \hline Product & Reynolds Averaged Expansion (Non-SGS Averages) \\
\hline$\left\langle\rho^{\prime}\right\rangle$ & $=0$ \\
$\langle f\rangle$ & $\neq \bar{f}$ \\
$\left\langle f^{\prime}\right\rangle$ & $\neq 0$ \\
$\langle\rho f\rangle$ & $=\tilde{\rho} \bar{f}$ \\
$\left\langle\rho f^{\prime}\right\rangle$ & $=0$ \\
$\langle\bar{f}\rangle$ & $=\bar{f}$ \\
$\langle\rho f g\rangle$ & $=\tilde{\rho}\left(\bar{f} \bar{g}+\overline{f^{\prime} g^{\prime}}\right)$ \\
$\left\langle\rho f^{\prime} g^{\prime}\right\rangle$ & $=\tilde{\rho} \overline{f^{\prime} g^{\prime}}$ \\
$\langle\rho f g h\rangle$ & $=\tilde{\rho}\left(\bar{f} \bar{g} \bar{h}+\bar{f} \overline{g^{\prime} h^{\prime}}+\bar{g} \overline{f^{\prime} h^{\prime}}+\bar{h} \overline{f^{\prime} g^{\prime}}+\overline{f^{\prime} g^{\prime} h^{\prime}}\right)$ \\
\hline
\end{tabular}

and

$$
\langle\rho \bar{f}\rangle=\langle\tilde{\rho} \bar{f}\rangle=\tilde{\rho} \bar{f}
$$

However, the LES convolution operator generally does not have these properties. Table 2 lists a convenient table of mass-weighted averages of products of various variables that do obey equations 11 and 12. The convolution operator produces additional terms that we will examine later, the so-called Leonard and cross terms.

Let us briefly consider non-mass-weighted variables. For example, $\tilde{f} \equiv\langle f\rangle$, and

$$
f=\bar{f}+f^{\prime}=\tilde{f}+f^{\prime \prime}
$$

Then

$$
\langle\rho f\rangle=\tilde{\rho} \bar{f}=\tilde{\rho} \tilde{f}+\overline{\rho^{\prime \prime} f^{\prime \prime}} .
$$

Table 3 summarizes the non-mass-weighted averages of products of various variables. Comparison with Table 2 shows why we prefer mass-weighted averaging: it eliminates density fluctuations from the mean flow equations, resulting in a significant simplification.

Germano notes that the algebra of the averaged equations may be simplified by introducing what he calls "generalized turbulent stresses," which may be further generalized to compressible flows as

$$
\tilde{\rho} \tau(f, g) \equiv\langle\rho f g\rangle-\tilde{\rho} \bar{f} \bar{g}=\tilde{\rho} \overline{f g}-\tilde{\rho} \bar{f} \bar{g}
$$


Table 3.

Non-Mass Weighted Averages of Products of Variables

\begin{tabular}{ll}
\hline \hline Product & Expansion (Non-SGS Averages) \\
\hline$\left\langle\rho^{\prime \prime}\right\rangle$ & $=\left\langle\rho^{\prime}\right\rangle=0$ \\
$\langle f\rangle$ & $=\tilde{f} \neq \bar{f}$ \\
$\left\langle f^{\prime \prime}\right\rangle$ & $=0 \neq\left\langle f^{\prime}\right\rangle$ \\
$\langle\rho f\rangle$ & $=\tilde{\rho} \tilde{f}+\overline{\rho^{\prime \prime} f^{\prime \prime}}$ \\
$\langle\tilde{f}\rangle$ & $=\tilde{f}$ \\
$\langle\rho f g\rangle$ & $=\tilde{\rho}\left(\tilde{f} \tilde{g}+\overline{f^{\prime \prime} g^{\prime \prime}}\right)+\tilde{f} \overline{\rho^{\prime \prime} g^{\prime \prime}}+\tilde{g} \overline{\rho^{\prime \prime} f^{\prime \prime}}+\overline{\rho^{\prime \prime} f^{\prime \prime} g^{\prime \prime}}$ \\
$\langle\rho f g h\rangle$ & $=\tilde{\rho}\left(\tilde{f} \tilde{g} \tilde{h}+\tilde{f} \overline{g^{\prime \prime} h^{\prime \prime}}+\tilde{g} \overline{f^{\prime \prime} h^{\prime \prime}}+\tilde{h} \overline{f^{\prime \prime} g^{\prime \prime}}+\overline{f^{\prime \prime} g^{\prime \prime} h^{\prime \prime}}\right)$ \\
& $+\tilde{g} \tilde{h} \overline{\rho^{\prime \prime} f^{\prime \prime}}+\tilde{f} \tilde{h} \overline{\rho^{\prime \prime} g^{\prime \prime}}+\tilde{f} \tilde{g} \overline{\rho^{\prime \prime} h^{\prime \prime}}$ \\
& $+\tilde{f} \overline{\rho^{\prime \prime} g^{\prime \prime} h^{\prime \prime}}+\tilde{g} \overline{\rho^{\prime \prime} f^{\prime \prime} h^{\prime \prime}}+\tilde{h} \overline{\rho^{\prime \prime} f^{\prime \prime} g^{\prime \prime}}+\overline{\rho^{\prime \prime} f^{\prime \prime} g^{\prime \prime} h^{\prime \prime}}$ \\
\hline
\end{tabular}

If $f$ and $g$ are both vectors, we shall assume $f g$ is the dyadic product unless otherwise noted. In the case of averages that satisfy equations 11 and 12, this generalized stress reduces to

$$
\tau(f, g)=\overline{f^{\prime} g^{\prime}}
$$

For LES filtering with a convolution operator, these relations are not satisfied in general, and the generalized stress may be expanded as

$$
\tau(f, g)=(\overline{\bar{f}} \bar{g}-\bar{f} \bar{g})+\left(\overline{f^{\prime} \bar{g}}+\overline{g^{\prime} \bar{f}}\right)+\overline{g^{\prime} f^{\prime}} .
$$

The terms on the right hand side are the familiar Leonard stresses, cross terms, and Reynolds stress. By not making this expansion just yet, we shall find that we can obtain transport equations that are invariant under the choice of averaging kernel, which will be useful in developing closure approximations.

We may also generalize equation 15 to the case of triple products:

$$
\begin{aligned}
& \tilde{\rho} \tau(f, g, h) \equiv\langle\rho f g h\rangle-\tilde{\rho} \bar{f} \bar{g} \bar{h}-\tilde{\rho}[\bar{f} \tau(g, h)+\bar{g} \tau(h, f)+\bar{h} \tau(f, g)] \\
& =\tilde{\rho}\left[\overline{\bar{f}} \bar{g} \bar{h}-\bar{f} \bar{g} \bar{h}-\bar{f}(\overline{\bar{g} \bar{h}}-\bar{g} \bar{h})-\bar{g}(\overline{\bar{h} \bar{f}}-\bar{h} \bar{f})-\bar{h}(\overline{\bar{f}} \bar{g}-\bar{f} \bar{g})-\overline{\bar{f}} \bar{g} h^{\prime}-\overline{\bar{f}} g^{\prime} \bar{h}-\overline{f^{\prime} \bar{g} \bar{h}}+\overline{f^{\prime} g^{\prime} h^{\prime}}\right] .
\end{aligned}
$$

If the averaging operation satisfies equations 11 and 12, this reduces to the triple correlation $\tau(f, g, h)=\overline{f^{\prime} g^{\prime} h^{\prime}}$.

Now, let us average equations 1 through 4:

$$
\frac{\partial \tilde{\rho}}{\partial t}+\nabla \cdot(\tilde{\rho} \overline{\mathbf{u}})=0
$$




$$
\begin{gathered}
\frac{\partial \tilde{\rho} \bar{f}}{\partial t}+\nabla \cdot[\tilde{\rho} \bar{f} \overline{\mathbf{u}}+\tilde{\rho} \tau(f, \mathbf{u})]=\left\langle S_{f}\right\rangle \\
\frac{\partial}{\partial t}[\tilde{\rho} \bar{f} \bar{g}+\tilde{\rho} \tau(f, g)]+\nabla \cdot\{\tilde{\rho}[\bar{f} \bar{g} \overline{\mathbf{u}}+\tau(f, g, \mathbf{u})+\bar{f} \tau(g, \mathbf{u})+\bar{g} \tau(\mathbf{u}, f)+\overline{\mathbf{u}} \tau(f, g)]\} \\
=\left\langle g S_{f}\right\rangle+\left\langle f S_{g}\right\rangle
\end{gathered}
$$

and

$$
\frac{\partial \tilde{\rho} \overline{\mathbf{u}}}{\partial t}+\nabla \cdot[\tilde{\rho} \overline{\mathbf{u}} \overline{\mathbf{u}}+\tilde{\rho} \tau(\mathbf{u}, \mathbf{u})]=\left\langle\mathbf{S}_{\mathbf{u}}\right\rangle .
$$

We can combine equations 19 and 20 (once for $\bar{f}$ and once for $\bar{g}$ ) to get

$$
\frac{\partial \tilde{\rho} \bar{f} \bar{g}}{\partial t}+\nabla \cdot(\tilde{\rho} \bar{f} \bar{g} \overline{\mathbf{u}})+\bar{g} \nabla \cdot[\tilde{\rho} \tau(f, \mathbf{u})]+\bar{f} \nabla \cdot[\tilde{\rho} \tau(g, \mathbf{u})]=\bar{g}\left\langle S_{f}\right\rangle+\bar{f}\left\langle S_{g}\right\rangle .
$$

Subtracting equation 23 from equation 21, we obtain the transport equation for the generalized second order stress,

$$
\begin{gathered}
\frac{\partial \tilde{\rho} \tau(f, g)}{\partial t}+\nabla \cdot[\tilde{\rho} \tau(f, g) \overline{\mathbf{u}}+\tilde{\rho} \tau(f, g, \mathbf{u})] \\
=\left\langle g S_{f}\right\rangle-\bar{g}\left\langle S_{f}\right\rangle+\left\langle f S_{g}\right\rangle-\bar{f}\left\langle S_{g}\right\rangle-\tilde{\rho} \tau(f, \mathbf{u}) \cdot \nabla \bar{g}-\tilde{\rho} \tau(g, \mathbf{u}) \cdot \nabla \bar{f}
\end{gathered}
$$

The same equations hold when we replace $\bar{g}$ by $\overline{\mathbf{u}}$, taking care with the dyadic product in the convection term. The average of equation 5 is

$$
\begin{gathered}
\frac{\partial}{\partial t}[\tilde{\rho} \bar{f} \overline{\mathbf{u}}+\tilde{\rho} \tau(f, \mathbf{u})]+\nabla \cdot\{\tilde{\rho}[\bar{f} \overline{\mathbf{u}} \overline{\mathbf{u}}+\tau(f, \mathbf{u}, \mathbf{u})+\bar{f} \tau(\mathbf{u}, \mathbf{u})+\tau(f, \mathbf{u}) \overline{\mathbf{u}}+\overline{\mathbf{u}} \tau(f, \mathbf{u})]\} \\
=\left\langle\mathbf{u} S_{f}\right\rangle+\left\langle f \mathbf{S}_{u}\right\rangle,
\end{gathered}
$$

We can combine equations 19, 20, and 22 to get

$$
\frac{\partial \tilde{\rho} \bar{f} \overline{\mathbf{u}}}{\partial t}+\nabla \cdot(\tilde{\rho} \bar{f} \overline{\mathbf{u}} \overline{\mathbf{u}})+\overline{\mathbf{u}} \nabla \cdot[\tilde{\rho} \tau(f, \mathbf{u})]+\bar{f} \nabla \cdot[\tilde{\rho} \tau(\mathbf{u}, \mathbf{u})]=\overline{\mathbf{u}}\left\langle S_{f}\right\rangle+\bar{f}\left\langle\mathbf{S}_{u}\right\rangle .
$$

Subtracting equation 26 from equation 25, we obtain the transport equation for the generalized second order flux,

$$
\begin{gathered}
\frac{\partial \tilde{\rho} \tau(f, \mathbf{u})}{\partial t}+\nabla \cdot[\tilde{\rho} \overline{\mathbf{u}} \tau(f, \mathbf{u})+\tilde{\rho} \tau(f, \mathbf{u}, \mathbf{u})] \\
=\left\langle\mathbf{u} S_{f}\right\rangle-\overline{\mathbf{u}}\left\langle S_{f}\right\rangle+\left\langle f \mathbf{S}_{u}\right\rangle-\bar{f}\left\langle\mathbf{S}_{u}\right\rangle-\tilde{\rho} \tau(f, \mathbf{u}) \cdot \nabla \overline{\mathbf{u}}-\tilde{\rho} \tau(\mathbf{u}, \mathbf{u}) \cdot \nabla \bar{f}
\end{gathered}
$$

By similar manipulations, we can derive a transport equation for each component of the generalized Reynolds stress, which is a special case of equation 24:

$$
\begin{gathered}
\frac{\partial \tilde{\rho} \tau\left(u_{i}, u_{j}\right)}{\partial t}+\nabla \cdot\left[\tilde{\rho} \tau\left(u_{i}, u_{j}\right) \overline{\mathbf{u}}+\tilde{\rho} \tau\left(u_{i}, u_{j}, \mathbf{u}\right)\right]+\tilde{\rho} \tau\left(u_{i}, \mathbf{u}\right) \cdot \nabla \bar{u}_{j}+\tilde{\rho} \tau\left(u_{j}, \mathbf{u}\right) \cdot \nabla \bar{u}_{i} \\
=\left\langle u_{i} S_{u_{j}}\right\rangle-\bar{u}_{i}\left\langle S_{u_{j}}\right\rangle+\left\langle u_{j} S_{u_{i}}\right\rangle-\bar{u}_{j}\left\langle S_{u_{i}}\right\rangle .
\end{gathered}
$$

This equation may be contracted to obtain the transport equation for the SGS kinetic energy, $\mathcal{K}=0.5 \operatorname{Tr}[\tau(\mathbf{u}, \mathbf{u})]=0.5(\langle\mathbf{u} \cdot \mathbf{u}\rangle-\overline{\mathbf{u}} \cdot \overline{\mathbf{u}})$,

$$
\frac{\partial \tilde{\rho} \mathcal{K}}{\partial t}+\nabla \cdot\left[\tilde{\rho} \mathcal{K} \overline{\mathbf{u}}+0.5 \tilde{\rho} \tau\left(u_{i}, u_{i}, \mathbf{u}\right)\right]+\tilde{\rho} \tau(\mathbf{u}, \mathbf{u}): \nabla \overline{\mathbf{u}}=\left\langle\mathbf{u} \cdot \mathbf{S}_{u}\right\rangle-\overline{\mathbf{u}} \cdot\left\langle\mathbf{S}_{u}\right\rangle .
$$

The summation convention applies to the triple correlation. 


\section{The Navier-Stokes Equations}

We assume that the details of turbulent flows can be described to a sufficiently high degree of accuracy by the multicomponent Navier-Stokes equations. We assume the fluid is a mixture of species described by the single velocity (mass weighted) representation.

Mass conservation is expressed by the continuity equation for each species $\alpha$ :

$$
\frac{\partial \rho_{\alpha}}{\partial t}+\nabla \cdot\left(\rho_{\alpha} \mathbf{u}\right)=-\nabla \cdot \mathbf{J}_{\alpha}+R_{\alpha}
$$

where $\rho_{\alpha}$ is the density of species $\alpha, t$ is time, $\mathbf{u}$ is the velocity, and $R_{\alpha}$ is the rate at which species $\alpha$ is created by chemical reactions. The exact diffusional mass flux is given implicitly by a complicated expression (for example, [13, 14, 15]; a clear summary of the governing equations and an efficient numerical algorithm for solving them are given by Ramshaw [16] and by Ramshaw and Chang [17]), but it will suffice for our present purpose to consider Fick's law,

$$
\mathbf{J}_{\alpha}=-\rho D \nabla\left(\rho_{\alpha} / \rho\right) \text {, }
$$

where $\rho$ is the total density, and $D$ is the species diffusivity. In addition, we shall assume that $D$ is independent of species. Equations 30 and 31 may be summed over species to obtain the total continuity equation

$$
\frac{\partial \rho}{\partial t}+\nabla \cdot(\rho \mathbf{u})=0
$$

The momentum equation is

$$
\frac{\partial(\rho \mathbf{u})}{\partial t}+\nabla \cdot(\rho \mathbf{u u})=\sum_{\alpha} \rho_{\alpha} \mathbf{F}_{\alpha}-\nabla P+\nabla \cdot \mathcal{T}
$$

where $\mathbf{F}_{\alpha}$ is the external body force per unit mass on species $\alpha$ and $P$ is the pressure. If $\mathbf{F}_{\alpha}=\mathbf{g}$ is the gravitational acceleration, the sum over forces reduces to the usual $\rho \mathbf{g}$. The viscous stress tensor $\mathcal{T}$ is given by

$$
\begin{gathered}
\mathcal{T}=\mu\left[\nabla \mathbf{u}+(\nabla \mathbf{u})^{T}\right]+\mu_{1} \nabla \cdot \mathbf{u} \mathcal{U} \\
=\mu\left[\nabla \mathbf{u}+(\nabla \mathbf{u})^{T}-\frac{2}{3} \nabla \cdot \mathbf{u} \mathcal{U}\right]+\mu_{\mathrm{b}} \nabla \cdot \mathbf{u} \mathcal{U} .
\end{gathered}
$$

Here $\mathcal{U}$ is the unit tensor, and $\mu$ is the coefficient of viscosity, $\mu_{1}$ is the second coefficient of viscosity, and $\mu_{\mathrm{b}}$ is the bulk viscosity. Comparing equation 7 with equation 33, we see that the equation for the kinetic energy density $K_{e}=0.5 \mathbf{u} \cdot \mathbf{u}$ is

$$
\frac{\partial\left(\rho K_{e}\right)}{\partial t}+\nabla \cdot\left(\rho K_{e} \mathbf{u}\right)=\sum_{\alpha} \rho_{\alpha} \mathbf{u} \cdot \mathbf{F}_{\alpha}-\mathbf{u} \cdot \nabla P+\mathbf{u} \cdot(\nabla \cdot \mathcal{T})
$$


We express energy conservation in terms of the specific thermal internal energy $I$ :

$$
\frac{\partial(\rho I)}{\partial t}+\nabla \cdot(\rho I \mathbf{u})=-P \nabla \cdot \mathbf{u}+\mathcal{T}: \nabla \mathbf{u}-\nabla \cdot \mathbf{q}+\sum_{\alpha} H_{\alpha} R_{\alpha}+\sum_{\alpha} \mathbf{F}_{\alpha} \cdot \mathbf{J}_{\alpha},
$$

where $\mathbf{q}$ is the diffusional heat flux, and $H_{\alpha}$ is the heat of formation of species $\alpha$. The final term vanishes when $\mathbf{F}_{\alpha}$ is the gravitational acceleration. The heat flux is another complicated function, and we choose to consider just the sum of Fourier's law and enthalpy diffusion:

$$
\mathbf{q}=-K \nabla T+\sum_{\alpha} h_{\alpha} \mathbf{J}_{\alpha}
$$

where $K$ is the multicomponent thermal conductivity, $h_{\alpha}$ is the enthalpy of species $\alpha$, and $T$ is the temperature. For our present purposes, the Dufour term may be neglected.

Although we have chosen to express energy conservation in terms of the thermal specific internal energy, there are several alternate possibilities. We could also use the total energy density $E=I+0.5(\mathbf{u} \cdot \mathbf{u})+\Phi$ or the enthalpy $h=I+P / \rho$. The corresponding equation for $E$ is

$$
\frac{\partial(\rho E)}{\partial t}+\nabla \cdot(\rho I \mathbf{u}+P \mathbf{u})=\nabla \cdot(\mathcal{T} \cdot \mathbf{u})-\nabla \cdot \mathbf{q}+\sum_{\alpha} H_{\alpha} R_{\alpha}+\sum_{\alpha} \mathbf{F}_{\alpha} \cdot \mathbf{J}_{\alpha}
$$

This equation is valid only if the potential energy $\Phi$ is independent of time. The term involving heats for formation can be eliminated by including the chemical energy in $I$, although that complicates the process of extracting $T$ from $I$. Other possibilities are summarized on page 562 of Bird, Stewart, and Lightfoot [14].

The constitutive relations can introduce considerable complexity into the problem of simulating turbulent flows. This complexity can be illustrated even for the relatively simple and frequently used case of the total pressure $P$ being the sum of ideal gas partial pressures:

$$
P=\sum_{\alpha} R \rho_{\alpha} T / M_{\alpha}
$$

where $R$ is the universal gas constant, and $M_{\alpha}$ is the molecular weight of species $\alpha$. The mixture internal energy is assumed to be given by

$$
\rho I=\sum_{\alpha} \rho_{\alpha} I_{\alpha}(T)
$$

where $I_{\alpha}$ is the species specific thermal internal energy. For combustion applications, the $I_{\alpha}$ are slightly nonlinear functions of $T$ that are usually taken from tables or polynomial fits to tables (for example, [18]).

Some applications may require modifications to the constitutive relations. For example, it is a good approximation to include radiative transfer in the interiors of most stars by 
the one-temperature gray diffusion approximation. In that case, the viscous stress tensor must be modified. If we include radiation in the gray diffusion approximation, the stress tensor is [19]

$$
\begin{gathered}
\mathcal{T}=\left(\mu+\mu_{\mathrm{r}}\right)\left[\nabla \mathbf{u}+(\nabla \mathbf{u})^{T}-\frac{2}{3} \nabla \cdot \mathbf{u} \mathcal{U}\right]+\left(\mu_{\mathrm{b}}+\frac{5 \mu_{\mathrm{r}}}{3}\right) \nabla \cdot \mathbf{u} \mathcal{U} \\
-\frac{1}{c^{2}}\left[\mathbf{u} \mathbf{q}_{\mathrm{r}}+\mathbf{q}_{\mathrm{r}} \mathbf{u}+\left(\mathbf{u} \cdot \mathbf{q}_{\mathrm{r}}\right) \mathcal{U}\right]
\end{gathered}
$$

where

$$
\mu_{\mathrm{r}}=4 a T^{4} / 15 c \kappa \rho
$$

is the radiative viscosity, $\kappa$ is the Rosseland mean opacity, $a$ is the Stefan-Boltzmann constant, $c$ is the speed of light, and

$$
\mathbf{q}_{\mathrm{r}}=-K_{\mathrm{r}} \nabla T=-\frac{4 a c T^{3}}{3 \kappa \rho} \nabla T
$$

is the radiative flux vector. In practice, the last line of equation 41 is usually neglected. However, the flux $\mathbf{q}_{\mathrm{r}}$ must be added to the ionic and electron heat flux defined in equation 37 because it is usually the dominant term.

The equation of state for the interior of most normal stars may be approximated by the sum of partial pressures for each species, with the ionic species treated as ideal gases:

$$
P=\sum_{\alpha} R \rho_{\alpha} T / M_{\alpha}+P_{e}+\frac{a T^{4}}{3},
$$

where $R$ is the universal gas constant. The electron pressure can be calculated from the usual degenerate gas equation of state [20], and the radiation pressure term assumes local thermodynamic equilibrium between the gas and radiation field. The relationship among temperature, density, internal energy, and composition is given by

$$
\rho I=\sum_{\alpha} \rho_{\alpha} I_{\alpha}(T)+\rho I_{e}+a T^{4}=\sum_{\alpha} \frac{R \rho_{\alpha} T}{M_{\alpha}\left(\gamma_{\alpha}-1\right)}+\rho I_{e}+a T^{4},
$$

where $I_{\alpha}$ is the ionic species specific thermal internal energy, $I_{e}$ is the electron internal energy, and $a$ is the radiation energy density constant. These thermal and caloric equations of state with $\gamma_{\alpha}=5 / 3$ are adequate for many stellar interiors and atmospheres simulations, but will have to be replaced for other applications, such as planetary interiors and the envelopes of low-mass stars. Other applications may require still other constitutive relations, but these two cases will serve as examples of the complexities that must be addressed in turbulence modeling of real gases. 


\section{The General Averaged Equations}

Let us begin by writing down the equations for the mean flow. Averaging equations 30, 32, 33 , and 36 , we obtain

$$
\begin{gathered}
\frac{\partial \tilde{\rho} \bar{Y}_{\alpha}}{\partial t}+\nabla \cdot\left[\tilde{\rho} \bar{Y}_{\alpha} \overline{\mathbf{u}}+\tilde{\rho} \tau\left(\mathbf{u}, Y_{\alpha}\right)+\left\langle\mathbf{J}_{\alpha}\right\rangle\right]=\left\langle R_{\alpha}\right\rangle, \\
\frac{\partial \tilde{\rho}}{\partial t}+\nabla \cdot(\tilde{\rho} \overline{\mathbf{u}})=0, \\
\frac{\partial(\tilde{\rho} \overline{\mathbf{u}})}{\partial t}+\nabla \cdot[\tilde{\rho} \overline{\mathbf{u}} \overline{\mathbf{u}}+\tilde{\rho} \tau(\mathbf{u}, \mathbf{u})-\langle\mathcal{T}\rangle]=\sum_{\alpha}\left\langle\rho_{\alpha} \mathbf{F}_{\alpha}\right\rangle-\nabla \tilde{P}=\tilde{\rho} \overline{\mathbf{g}}-\nabla \tilde{P},
\end{gathered}
$$

and

$$
\begin{aligned}
& \frac{\partial(\tilde{\rho} \bar{I})}{\partial t}+\nabla \cdot\left[\tilde{\rho} \bar{I} \overline{\mathbf{u}}+\tilde{\rho} \tau(I, \mathbf{u})-\langle K \nabla T\rangle+\sum_{\alpha}\left\langle h_{\alpha} \mathbf{J}_{\alpha}\right\rangle\right] \\
& =\sum_{\alpha} H_{\alpha}\left\langle R_{\alpha}\right\rangle-\langle P \nabla \cdot \mathbf{u}\rangle+\langle\mathcal{T}: \nabla \mathbf{u}\rangle+\sum_{\alpha}\left\langle\mathbf{F}_{\alpha} \cdot \mathbf{J}_{\alpha}\right\rangle .
\end{aligned}
$$

Here $Y_{\alpha}=\rho_{\alpha} / \rho$ is the mass fraction of species $\alpha$. In equation 48, we have included the

special case of $\mathbf{F}_{\alpha}=\mathbf{g}$. The last term in equation 49 vanishes in this case. We shall assume gravity is the only body force in the remainder of this report. Note that up to this point the equations are exact. However, we now have a serious closure problem because there are several unknown turbulence correlations that appear in these equations.

We can derive transport equations for some of these unknown terms. The primary turbulent correlations are the turbulence kinetic energy density $\mathcal{K}$ and the generalized Reynolds stress tensor $\tau(\mathbf{u}, \mathbf{u})$. Expanding the source terms in equation 29, we obtain

$$
\begin{gathered}
\frac{\partial \tilde{\rho} \mathcal{K}}{\partial t}+\nabla \cdot\left[\tilde{\rho} \mathcal{K} \overline{\mathbf{u}}+0.5 \tilde{\rho} \tau\left(u_{i}, u_{i}, \mathbf{u}\right)\right]+\tilde{\rho} \tau(\mathbf{u}, \mathbf{u}): \nabla \overline{\mathbf{u}} \\
=\langle\rho \mathbf{g} \cdot \mathbf{u}\rangle-\tilde{\rho} \overline{\mathbf{g}} \cdot \overline{\mathbf{u}}-[\langle\mathbf{u} \cdot \nabla P\rangle-\overline{\mathbf{u}} \cdot \nabla \tilde{P}]+[\langle\mathbf{u} \cdot(\nabla \cdot \mathcal{T})\rangle-\overline{\mathbf{u}} \cdot(\nabla \cdot\langle\mathcal{T}\rangle)] .
\end{gathered}
$$

Similarly, the transport equation for each component of the generalized Reynolds stress is

$$
\begin{gathered}
\frac{\partial}{\partial t} \tilde{\rho} \tau\left(u_{j}, u_{i}\right)+\nabla \cdot\left[\tilde{\rho} \tau\left(u_{j}, u_{i}\right) \overline{\mathbf{u}}+\tilde{\rho} \tau\left(u_{j}, u_{i}, \mathbf{u}\right)\right]+\tilde{\rho} \tau\left(u_{i}, \mathbf{u}\right) \cdot \nabla \bar{u}_{j}+\tilde{\rho} \tau\left(u_{j}, \mathbf{u}\right) \cdot \nabla \bar{u}_{i} \\
=\left\langle\rho u_{j} g_{i}\right\rangle+\left\langle\rho u_{i} g_{j}\right\rangle-\tilde{\rho}\left(\bar{u}_{j} \bar{g}_{i}+\bar{u}_{i} \bar{g}_{j}\right)-\left\langle u_{j}(\nabla P)_{i}\right\rangle-\left\langle u_{i}(\nabla P)_{j}\right\rangle+\bar{u}_{j}(\nabla \tilde{P})_{i}+\bar{u}_{i}(\nabla \tilde{P})_{j} \\
+\left\langle u_{j}(\nabla \cdot \mathcal{T})_{i}\right\rangle-\bar{u}_{j}(\nabla \cdot\langle\mathcal{T}\rangle)_{i}+\left\langle u_{i}(\nabla \cdot \mathcal{T})_{j}\right\rangle-\bar{u}_{i}(\nabla \cdot\langle\mathcal{T}\rangle)_{j} .
\end{gathered}
$$

This equation may be contracted to obtain equation 50 .

We can derive a transport equation for the generalized turbulent species mass flux $\tau\left(Y_{\alpha}, \mathbf{u}\right)$ by taking $f=Y_{\alpha}$ in equation 27 . The result is

$$
\frac{\partial \tilde{\rho} \tau\left(Y_{\alpha}, \mathbf{u}\right)}{\partial t}+\nabla \cdot\left[\tilde{\rho} \overline{\mathbf{u}} \tau\left(Y_{\alpha}, \mathbf{u}\right)+\tilde{\rho} \tau\left(Y_{\alpha}, \mathbf{u}, \mathbf{u}\right)\right]+\tilde{\rho} \tau(\mathbf{u}, \mathbf{u}) \cdot \nabla \bar{Y}_{\alpha}+\tilde{\rho} \tau\left(Y_{\alpha}, \mathbf{u}\right) \cdot \nabla \overline{\mathbf{u}}=
$$


$\left\langle\rho Y_{\alpha} \mathbf{g}\right\rangle-\tilde{\rho} \bar{Y}_{\alpha} \overline{\mathbf{g}}+\left\langle\mathbf{u} R_{\alpha}\right\rangle-\overline{\mathbf{u}}\left\langle R_{\alpha}\right\rangle-\left\langle\mathbf{u} \nabla \cdot \mathbf{J}_{\alpha}\right\rangle+\overline{\mathbf{u}} \nabla \cdot\left\langle\mathbf{J}_{\alpha}\right\rangle+\left\langle Y_{\alpha} \nabla \cdot \mathcal{T}\right\rangle-\bar{Y}_{\alpha} \nabla \cdot\langle\mathcal{T}\rangle-\left\langle Y_{\alpha} \nabla P\right\rangle+\bar{Y}_{\alpha} \nabla \tilde{P}$.

We also require the transport equation for the turbulent heat flux $\tau(I, \mathbf{u})$, which is

$$
\begin{aligned}
& \frac{\partial \tilde{\rho} \tau(I, \mathbf{u})}{\partial t}+\nabla \cdot[\tilde{\rho} \overline{\mathbf{u}} \tau(I, \mathbf{u})+\nabla \cdot \tilde{\rho} \tau(I, \mathbf{u}, \mathbf{u})]+\tilde{\rho} \tau(I, \mathbf{u}) \cdot \nabla \overline{\mathbf{u}}+\tilde{\rho} \tau(\mathbf{u}, \mathbf{u}) \cdot \nabla \bar{I} \\
&=\langle\rho I \mathbf{g}\rangle-\tilde{\rho} \bar{I} \overline{\mathbf{g}}-\langle I \nabla P\rangle+\bar{I} \nabla \tilde{P}-\langle\mathbf{u} P \nabla \cdot \mathbf{u}\rangle+\overline{\mathbf{u}}\langle P \nabla \cdot \mathbf{u}\rangle+\langle I \nabla \cdot \mathcal{T}\rangle-\bar{I} \nabla \cdot\langle\mathcal{T}\rangle \\
&+\langle\mathbf{u} \nabla \cdot K \nabla T\rangle-\overline{\mathbf{u}} \nabla \cdot\langle K \nabla T\rangle+\langle\mathbf{u}(\mathcal{T}: \nabla \mathbf{u})\rangle-\overline{\mathbf{u}}\langle\mathcal{T}: \nabla \mathbf{u}\rangle \\
&+\sum_{\alpha}\left[H_{\alpha}\left(\left\langle R_{\alpha} \mathbf{u}\right\rangle-\left\langle R_{\alpha}\right\rangle \overline{\mathbf{u}}\right)-\left\langle\mathbf{u} \nabla \cdot\left(h_{\alpha} \mathbf{J}_{\alpha}\right)\right\rangle+\overline{\mathbf{u}} \nabla \cdot\left\langle h_{\alpha} \mathbf{J}_{\alpha}\right\rangle\right] .
\end{aligned}
$$

In both equations 52 and 53, the gravitational terms drop out when $\mathbf{g}$ is a constant.

Next we need the second order correlations for products of the scalars. Let us begin with the generalized density fluctuation scale, $\tau^{\prime}(\rho, \rho) \equiv\langle\rho \rho\rangle-\tilde{\rho} \tilde{\rho}$, which is equal to the root-mean-square (rms) density fluctuation $\overline{\rho^{\prime} \rho^{\prime}}$ for averages that obey equations 11 and 12 . It is easy to show that

$$
\frac{\partial \rho^{2}}{\partial t}+\nabla \cdot\left(\rho^{2} \mathbf{u}\right)=-\rho^{2} \nabla \cdot \mathbf{u}
$$

by manipulating the continuity equation 32 . We can perform analogous operations on equation 47 for the mean flow, and subtract the result from the average of equation 54 to obtain

$$
\frac{\partial \tau^{\prime}(\rho, \rho)}{\partial t}+\nabla \cdot\left[\tau^{\prime}(\rho, \rho) \overline{\mathbf{u}}+\left\langle\rho^{2} \mathbf{u}\right\rangle-\left\langle\rho^{2}\right\rangle \overline{\mathbf{u}}\right]=-\left\langle\rho^{2} \nabla \cdot \mathbf{u}\right\rangle+\tilde{\rho}^{2} \nabla \cdot \overline{\mathbf{u}} .
$$

Other potentially useful correlations are $\tau\left(Y_{\alpha}, Y_{\beta}\right), \tau\left(I, Y_{\alpha}\right)$, and $\tau(I, I)$. Their transport equations are

$$
\begin{gathered}
\frac{\partial \tilde{\rho} \tau\left(Y_{\alpha}, Y_{\beta}\right)}{\partial t}+\nabla \cdot\left[\tilde{\rho} \tau\left(Y_{\alpha}, Y_{\beta}\right) \overline{\mathbf{u}}+\tilde{\rho} \tau\left(Y_{\alpha}, Y_{\beta}, \mathbf{u}\right)\right]+\tilde{\rho} \tau\left(Y_{\alpha}, \mathbf{u}\right) \cdot \nabla \bar{Y}_{\beta}+\tilde{\rho} \tau\left(Y_{\beta}, \mathbf{u}\right) \cdot \nabla \bar{Y}_{\alpha} \\
=\left\langle Y_{\beta} R_{\alpha}\right\rangle-\bar{Y}_{\beta}\left\langle R_{\alpha}\right\rangle+\left\langle Y_{\alpha} R_{\beta}\right\rangle-\bar{Y}_{\alpha}\left\langle R_{\beta}\right\rangle-\left\langle Y_{\beta} \nabla \cdot \mathbf{J}_{\alpha}\right\rangle+\bar{Y}_{\beta} \nabla \cdot\left\langle\mathbf{J}_{\alpha}\right\rangle-\left\langle Y_{\alpha} \nabla \cdot \mathbf{J}_{\beta}\right\rangle+\bar{Y}_{\alpha} \nabla \cdot\left\langle\mathbf{J}_{\beta}\right\rangle, \quad(56) \\
\frac{\partial \tilde{\rho} \tau\left(I, Y_{\alpha}\right)}{\partial t}+\nabla \cdot\left[\tilde{\rho} \tau\left(I, Y_{\alpha}\right) \overline{\mathbf{u}}+\tilde{\rho} \tau\left(I, Y_{\alpha}, \mathbf{u}\right)\right]+\tilde{\rho} \tau(I, \mathbf{u}) \cdot \nabla \bar{Y}_{\alpha}+\tilde{\rho} \tau\left(Y_{\alpha}, \mathbf{u}\right) \cdot \nabla \bar{I} \\
=\left\langle I R_{\alpha}\right\rangle-\bar{I}\left\langle R_{\alpha}\right\rangle-\left\langle I \nabla \cdot \mathbf{J}_{\alpha}\right\rangle+\bar{I} \nabla \cdot\left\langle\mathbf{J}_{\alpha}\right\rangle-\left\langle Y_{\alpha} P \nabla \cdot \mathbf{u}\right\rangle+\bar{Y}_{\alpha}\langle P \nabla \cdot \mathbf{u}\rangle \\
\quad+\left\langle Y_{\alpha} \mathcal{T}: \nabla \mathbf{u}\right\rangle-\bar{Y}_{\alpha}\langle\mathcal{T}: \nabla \mathbf{u}\rangle+\left\langle Y_{\alpha} \nabla \cdot(K \nabla T)\right\rangle-\bar{Y}_{\alpha} \nabla \cdot\langle K \nabla T\rangle \\
\quad+\sum_{\beta}\left[\left\langle Y_{\alpha} H_{\beta} R_{\beta}\right\rangle-\bar{Y}_{\alpha}\left\langle H_{\beta} R_{\beta}\right\rangle-\left\langle Y_{\alpha} \nabla \cdot\left(h_{\beta} \mathbf{J}_{\beta}\right)\right\rangle+\bar{Y}_{\alpha} \nabla \cdot\left\langle h_{\beta} \mathbf{J}_{\beta}\right\rangle\right]
\end{gathered}
$$

and

$$
\begin{gathered}
\frac{\partial \tilde{\rho} \tau(I, I)}{\partial t}+\nabla \cdot[\tilde{\rho} \tau(I, I) \overline{\mathbf{u}}+\tilde{\rho} \tau(I, I, \mathbf{u})]+2 \tilde{\rho} \tau(I, \mathbf{u}) \cdot \nabla \bar{I} \\
=-2\langle I P \nabla \cdot \mathbf{u}\rangle+2 \bar{I}\langle P \nabla \cdot \mathbf{u}\rangle+2\langle I \mathcal{T}: \nabla \mathbf{u}\rangle-2 \bar{I}\langle\mathcal{T}: \nabla \mathbf{u}\rangle+2\langle I \nabla \cdot(K \nabla T)\rangle-2 \bar{I} \nabla \cdot\langle K \nabla T\rangle \\
+2 \sum_{\alpha}\left[\left\langle I H_{\alpha} R_{\alpha}\right\rangle-\bar{I}\left\langle H_{\alpha} R_{\alpha}\right\rangle-\left\langle I \nabla \cdot\left(h_{\alpha} \mathbf{J}_{\alpha}\right)\right\rangle+\bar{I} \nabla \cdot\left\langle h_{\alpha} \mathbf{J}_{\alpha}\right\rangle\right]
\end{gathered}
$$




\section{Comments on Closure Approximations}

Equations 46 through 49 are to be solved for the mean flow variables. However, this can be done only after specifying $\tau(\mathbf{u}, \mathbf{u}), \tau(I, \mathbf{u}), \tau\left(Y_{\alpha}, \mathbf{u}\right),\left\langle\mathbf{J}_{\alpha}\right\rangle,\left\langle R_{\alpha}\right\rangle,\langle K \nabla T\rangle,\left\langle h_{\alpha} \mathbf{J}_{\alpha}\right\rangle,\langle P \nabla \cdot \mathbf{u}\rangle$, $\langle\mathcal{T}: \nabla \mathbf{u}\rangle, \tilde{P}$, and $\langle\mathcal{T}\rangle$. If the external force is anything other than a constant gravitational field, it may be necessary to evaluate additional unknown correlations. My purpose here is not to propose closure approximations for these turbulence functionals, but to point out some issues that must be addressed.

The turbulence functionals fall into several broad groups according to their mathematical form and the kinds of approximations must be used to model them. The most basic are the averaged constitutive relations: the thermal and caloric equations of state and the transport terms. We now discuss some examples that range from the simplest ideal gas models to the real-gas physics used in combustion studies. These examples illustrate some issues that are often ignored by turbulence models.

The constitutive relations may or may not cause problems under averaging, depending on their complexity. The simplest case is an ideal gas with constant ratio of specific heats $\gamma$, mean molecular weight $M$, constant specific heat at constant volume $C_{v}$, and no chemical inhomogeneities. The averaged pressure is then

$$
\tilde{P}=\langle(\gamma-1) \rho I\rangle=(\gamma-1) \tilde{\rho} \bar{I}
$$

and the averaged caloric equation of state is

$$
\langle\rho I\rangle=\tilde{\rho} \bar{I}=\left\langle C_{v} \rho T\right\rangle=C_{v} \tilde{\rho} \bar{T}
$$

In this case, only known mean flow quantities are involved, and things are simple.

For many of our applications, the thermal equation of state is the sum of partial pressures of ideal gases:

$$
P=\sum_{\alpha} \frac{R}{M_{\alpha}} \rho Y_{\alpha} T .
$$

If the mean molecular weight $M=\left(\sum_{\alpha} Y_{\alpha} / M_{\alpha}\right)^{-1}$ is constant, then the averaged pressure is

$$
\tilde{P}=R \tilde{\rho} \bar{T} / M
$$

In the more general case, the averaged pressure is

$$
\tilde{P}=\sum_{\alpha} \frac{R}{M_{\alpha}} \tilde{\rho}\left[\bar{Y}_{\alpha} \bar{T}+\tau\left(Y_{\alpha}, T\right)\right]=\sum_{\alpha} \frac{R}{M_{\alpha}} \tilde{\rho}\left[\bar{Y}_{\alpha} \bar{T}+\left(\overline{\bar{Y}}{ }_{\alpha} \bar{T}-\bar{Y}_{\alpha} \bar{T}\right)+\overline{Y_{\alpha}^{\prime} \bar{T}}+\overline{\bar{Y}_{\alpha} T^{\prime}}+\overline{Y_{\alpha}^{\prime} T^{\prime}}\right] .
$$


We get Leonard, cross, and second-order correlation terms for LES convolutions, and the second order correlation survives in the other types of averages as well. ${ }^{3}$ It is not clear how to model these, and we normally ignore all but the first term in practice. Solving equation 57 for the correlation is not an attractive prospect. The situation gets even more difficult with real-gas equations of state, such as for the degenerate electron gas and radiation pressure in stellar interiors.

There is a similar problem with the caloric equation of state that relates internal energy to temperature. The simplest case is the combustion model used in the COYOTE program,

$$
\rho I=\sum_{\alpha} \rho Y_{\alpha} I_{\alpha}(T)
$$

where $I_{\alpha}(T)$ is a piecewise linear interpolating function in $T$ taken from the JANAF tables [18] and is therefore mildly nonlinear. A common practice in combustion research is to fit the JANAF tables with a sixth degree polynomial. In such cases, the convolution process leads to unknown correlations that are made all the more intractable by the nonlinear relations between the $I_{\alpha}$ and $T$ :

$$
\tilde{\rho} \bar{I}=\sum_{\alpha} \tilde{\rho} \overline{Y_{\alpha} I_{\alpha}(T)} \neq \sum_{\alpha} \tilde{\rho} \bar{Y}_{\alpha} I_{\alpha}(\bar{T})
$$

However, the right-hand side of equation 65 is usually used in practice to compute $\bar{T}$ in terms of $\bar{I}$. However, note that the $\bar{T}$ so derived is not quite the same as $\langle\rho T\rangle / \tilde{\rho}$ due to the neglect of certain turbulence correlation terms, even when each species has a constant specific heat at constant volume.

Another difficulty arises in recovering the temperature from $E$ if a total energy equation is used.

$$
\begin{aligned}
\bar{\rho} \tilde{E} & =\langle\rho E\rangle \\
& =0.5\langle\rho \mathbf{u} \cdot \mathbf{u}\rangle+\langle\rho I\rangle+\langle\rho \Phi\rangle \\
& =0.5 \tilde{\rho} \overline{\mathbf{u}} \cdot \overline{\mathbf{u}}+\tilde{\rho} \mathcal{K}+\tilde{\rho} \bar{I}+\tilde{\rho} \bar{\Phi}
\end{aligned}
$$

Even neglecting the potential energy term, one has the problem of not only still having the aforementioned difficulty of converting $\bar{I}$ into $\bar{T}$, but also needing accurate values of the generalized Reynolds stress or $\mathcal{K}$. Unfortunately, these are not usually known to better than a few percent. Furthermore, there are truncation errors of unknown magnitude in $\overline{\mathbf{u}}$, which can lead to substantial errors in $\bar{T}$. This can be disastrous in simulations where it is essential to have accurate temperatures, such as buoyancy-driven flows and chemically reactive flows.

\footnotetext{
${ }^{3}$ One must be mindful of constraints on the mass fractions. It is easy to show that $\sum_{\alpha} Y_{\alpha}=\sum_{\alpha} \bar{Y}_{\alpha}=1$ and ${\overline{Y^{\prime}}}_{\alpha}=\sum_{\alpha}{\overline{Y^{\prime}}}_{\alpha}=\sum_{\alpha} Y_{\alpha}^{\prime}=\sum_{\alpha} \tau\left(Y_{\alpha}, T\right)=0$. However, the sum in equation 63 is weighted by $M_{\alpha}^{-1}$ and does not reduce to any simple value.
} 
The $P d V$ work term in the mean-flow energy equation introduces another problem, which occurs even in the case of a simple ideal gas with a constant $\gamma$ :

$$
\langle P \nabla \cdot \mathbf{u}\rangle=(\gamma-1)\langle\rho I \nabla \cdot \mathbf{u}\rangle=\tilde{P} \nabla \cdot \overline{\mathbf{u}}+(\gamma-1) \tilde{\rho} \tau(I, \nabla \cdot \mathbf{u}) .
$$

Once again, the first term on the right-hand side is usually all that is retained in practice. Going to a total energy equation does not help. The $P \mathbf{u}$ term in the advective fluxes has exactly the same problem, which can be seen by simply deleting the divergence operators from equation 67 .

Next let us consider the molecular transport terms. In most engineering applications $\mu$ and $K$ are assumed constant or so weakly dependent on $\rho_{\alpha}$ and $T$ that, for example,

$$
\langle K \nabla T\rangle \simeq\left\langle K\left(\rho_{\alpha}, T\right)\right\rangle \nabla \bar{T} \simeq K\left(\tilde{\rho}_{\alpha}, \bar{T}\right) \nabla \bar{T}
$$

These kinds of approximations immediately lead to simplifications in the mean flow equations, but cannot be justified in all cases. Indeed, Deupree [21] has shown that equation 68 is of dubious validity in stellar envelopes. The situation still isn't simple even in the case of constant $K$ because the gradient operates on the non-mass-weighted mean temperature $\tilde{T}$, not $\bar{T}$. However, the approximation in equation 68 is almost always used in practice. Similar considerations apply to $\langle\mathcal{T}\rangle,\left\langle\mathbf{J}_{\alpha}\right\rangle,\left\langle h_{\alpha} \mathbf{J}_{\alpha}\right\rangle$, and $\langle\mathcal{T}: \nabla \mathbf{u}\rangle$. Even in the case of constant viscosity $\mu$, for example, $\langle\mathcal{T}\rangle$ contains derivatives of $\tilde{u}$, not $\bar{u}$. In general, $\mu$ will depend at least on $T$, so correlations between velocity and functions of thermodynamic variables appear.

A similar situation exists for the chemical reaction terms. This is a serious problem in turbulent combustion theory where turbulent rates are typically quite different than those given by the laminar rate formulas using mean flow quantities (for example, [4, 22]). The complexity of these terms can be seen in Appendix C. The influence of turbulence on reaction rates is one of the major unsolved problems in combustion theory and will not be addressed further in this report.

Next let us consider the generalized stresses $\tau(\mathbf{u}, \mathbf{u}), \tau(I, \mathbf{u})$, and $\tau\left(Y_{\alpha}, \mathbf{u}\right)$. In principle, we could solve the transport equations derived in the previous section. These equations introduce the additional correlations $\tau\left(u_{i}, u_{j}, \mathbf{u}\right),\langle\mathbf{u} \nabla P\rangle,\langle\mathbf{u} \nabla \cdot \mathcal{T}\rangle, \tau\left(Y_{\alpha}, \mathbf{u}, \mathbf{u}\right),\left\langle\mathbf{u} R_{\alpha}\right\rangle$, $\left\langle\mathbf{u} \nabla \cdot \mathbf{J}_{\alpha}\right\rangle,\left\langle\mathbf{u} \nabla \cdot\left(h_{\alpha} \mathbf{J}_{\alpha}\right)\right\rangle,\left\langle Y_{\alpha} \nabla \cdot \mathcal{T}\right\rangle,\left\langle Y_{\alpha} \nabla P\right\rangle, \tau(I, \mathbf{u}, \mathbf{u}),\langle I \nabla P\rangle,\langle\mathbf{u} P \nabla \cdot \mathbf{u}\rangle,\langle I \nabla \cdot \mathcal{T}\rangle$, $\langle\mathbf{u} \nabla \cdot(K \nabla T)\rangle$, and $\langle\mathbf{u}(\mathcal{T}: \nabla \mathbf{u})\rangle$. It is possible to derive equations for these correlations, but still more unknown correlations would appear. To close the system of equations, we must make some closure approximations that relate these correlations to mean flow variables and to other correlations in the system.

In addition to the closure problem, there may be large computational costs in solving transport equations for $\tau(I, \mathbf{u})$ and $\tau\left(Y_{\alpha}, \mathbf{u}\right)$. If there are $N$ species, there are $3(N+1)$ scalar transport equations to be solved. Clearly, simple algebraic closure models are desirable. 
In practice, $\tau(\mathbf{u}, \mathbf{u}), \tau(I, \mathbf{u})$, and $\tau\left(Y_{\alpha}, \mathbf{u}\right)$ often are approximated algebraically. It is important that any such approximations maintain the original Galilean and tensor invariance of the original equations. There may be realizability conditions that must be met as well, such as $\mathcal{K}$ must be non-negative and $\tau(\mathbf{u}, \mathbf{u})$ must be symmetric.

The most fundamental correlation that must be calculated is the generalized Reynolds stress tensor. For turbulence models that do not solve the transport equation for the components of the Reynolds stress, a commonly used approximation is

$$
\tilde{\rho} \tau(\mathbf{u}, \mathbf{u})=\frac{2 \tilde{\rho} \mathcal{K}}{3} \mathcal{U}-\mu_{t}\left[\nabla \overline{\mathbf{u}}+(\nabla \overline{\mathbf{u}})^{T}-\frac{2}{3} \nabla \cdot \overline{\mathbf{u}} \mathcal{U}\right],
$$

where $\mu_{t}$ is the eddy viscosity coefficient. There are numerous models in the literature for evaluating the eddy viscosity, ranging from a simple constant to the baroque (for example, Horiuti [23] discusses LES closures that involve quantities that have been filtered as many as 4 times!). A commonly used formalism is the Smagorinsky model [24], which is an algebraic model that evaluates $\mu_{t}$ from the gradient of the mean flow velocity. The turbulent pressure, the first term on the right-hand side of equation 69 , is usually absorbed into the total pressure for incompressible flows. For compressible flows, a common approach is to introduce a transport equation for $\mathcal{K}$ and assume

$$
\mu_{t}=C_{\mu} L \mathcal{K}^{1 / 2}
$$

where $C_{\mu}$ is a constant and $L$ is a turbulence length scale. Introduction of at least this one transport equation is important in the simulation of flows with rapidly changing conditions, such as in an internal combustion engine, since production and decay processes may be far from equilibrium. We shall not address this problem further in this report.

The final class of correlations that we shall mention is the $\tau(f, \mathbf{u})$. These almost always are modeled with the gradient-flux approximation. This approximation assumes

$$
\tau(f, \mathbf{u})=-\frac{\mu_{t}}{\tilde{\rho} \mathrm{Sc}_{f}} \nabla \bar{f}
$$

where $\mathrm{Sc}_{f}$ is the appropriate turbulent Schmidt number. It is well-known that this approximation is often seriously in error and can even have the wrong sign [25].

Obviously, this discussion only scratches the surface of the closure problem. For example, the $\tau(f, \mathbf{u})$ for LES convolutions can be expanded into a sum of Leonard, cross, and second-order fluctuation terms, each of which may need to be modeled in its own way rather than lumping all three terms into a gradient-flux approximation. Again, this subject is beyond the scope of this report. 


\section{Summary and Concluding Remarks}

We have generalized the incompressible equations developed by Germano to describe compressible flows with mass-weighted averages. Transport equations for all of the generalized second order correlations were derived. Since we used a very general averaging procedure, these equations compose a basic, internally consistent, foundation for future research and development of single-point statistical turbulence transport models for fluids in the continuum approximation, including the well-known $k-\epsilon$ model and most LES models. All closure approximations must be consistent with these equations and their underlying symmetries and realizability conditions.

We note that the equations derived here unify several different types of turbulence modeling. Germano noted the averaging invariance of the transport equations for the general class of averages that can be expressed in terms of an averaging kernel $G$, and this universality of the form of the transport equations suggests that closure approximations may in some sense also be invariant or only weakly dependent on the type of average. We concur and note that this suggestion applies to compressible flows as well as incompressible. We further note that this observation is consistent with the widespread use of dimensional analysis to generate closure approximations, which constrains the form of the closure regardless of the details of the averaging operator. The transport equations should be useful in developing improved algebraic closure models, or at least in constraining the types of models considered.

Finally, we discussed some frequently ignored complications that occur in compressible statistical turbulence models. These difficulties arise even in such fundamental quantities as the averaged pressure $\tilde{P}$ and the averaged $P d V$ work term. To our knowledge, the errors incurred by neglecting the various correlations in the equations of state and $P d V$ term have never been evaluated. This would be a useful area for future research.

I hope it is clear that the whole enterprise of treating turbulence in terms of some kind of average is neither clean nor elegant. It is disconcerting to ponder the multitude of unpleasantly complicated functionals that must be approximated regardless of which averaging kernel is chosen. While some models have achieved modest success as useful engineering approximations, it seems there ought to be a better approach. Perhaps this report will inspire someone to search for it.

\section{Acknowledgements}

I thank John Ramshaw for several useful discussions. This work was performed under the auspices of the U. S. Department of Energy by the Lawrence Livermore National Laboratory under contract number W-7405-ENG-48. 


\section{A Useful Vector and Tensor Relations}

Most work in hydrodynamics is done in Cartesian, cylindrical, or spherical coordinates, so we give the basic vector operators in these three systems. The gradient of a scalar is

$$
\nabla P= \begin{cases}\frac{\partial P}{\partial x} \hat{x}+\frac{\partial P}{\partial y} \hat{y}+\frac{\partial P}{\partial z} \hat{z} & \text { Cartesian } \\ \frac{\partial P}{\partial r} \hat{r}+\frac{1}{r} \frac{\partial P}{\partial \theta} \hat{\theta}+\frac{\partial P}{\partial z} \hat{z} & \text { Cylindrical } \\ \frac{\partial P}{\partial r} \hat{r}+\frac{1}{r} \frac{\partial P}{\partial \theta} \hat{\theta}+\frac{1}{r \sin \theta} \frac{\partial P}{\partial \phi} \hat{\phi} & \text { Spherical }\end{cases}
$$

The divergence of a vector is

$$
\nabla \cdot \mathbf{u}= \begin{cases}\frac{\partial u}{\partial x}+\frac{\partial v}{\partial y}+\frac{\partial w}{\partial z} & \text { Cartesian } \\ \frac{1}{r} \frac{\partial r u}{\partial r}+\frac{1}{r} \frac{\partial v}{\partial \theta}+\frac{\partial w}{\partial z} & \text { Cylindrical } \\ \frac{1}{r^{2}} \frac{\partial r^{2} u}{\partial r}+\frac{1}{r \sin \theta} \frac{\partial(v \sin \theta)}{\partial \theta}+\frac{1}{r \sin \theta} \frac{\partial w}{\partial \phi} & \text { Spherical }\end{cases}
$$

The curl of a vector may be written as a determinant.

$$
\nabla \times \mathbf{F}=\frac{1}{h_{1} h_{2} h_{3}}\left|\begin{array}{ccc}
h_{1} \hat{e}_{1} & h_{2} \hat{e}_{2} & h_{3} \hat{e}_{3} \\
\partial / \partial x_{1} & \partial / \partial x_{2} & \partial / \partial x_{3} \\
h_{1} F_{1} & h_{2} F_{2} & h_{3} F_{3}
\end{array}\right|,
$$

where $h_{i}$ is unity except $h_{2}=r$ in cylindrical coordinates, and $h_{\theta}=h_{2}=r$ and $h_{\phi}=h_{3}=$ $r \sin \theta$ in spherical coordinates.

We also deal with tensors, most of which are either symmetric or dyadics. In general, a rank two tensor $\mathcal{T}$ may be represented by a matrix:

$$
\mathcal{T}=\left[\begin{array}{lll}
\tau_{11} & \tau_{12} & \tau_{13} \\
\tau_{21} & \tau_{22} & \tau_{23} \\
\tau_{31} & \tau_{32} & \tau_{33}
\end{array}\right]
$$

The dyadic uv may be written

$$
\mathbf{u v}=\left[\begin{array}{lll}
u_{1} v_{1} & u_{1} v_{2} & u_{1} v_{3} \\
u_{2} v_{1} & u_{2} v_{2} & u_{2} v_{3} \\
u_{3} v_{1} & u_{3} v_{2} & u_{3} v_{3}
\end{array}\right]
$$

One of the more commonly encountered tensors is the gradient of the velocity vector.

$$
\begin{gathered}
\nabla \mathbf{u}=\left[\begin{array}{lll}
\partial u / \partial x & \partial v / \partial x & \partial w / \partial x \\
\partial u / \partial y & \partial v / \partial y & \partial w / \partial y \\
\partial u / \partial z & \partial v / \partial z & \partial w / \partial z
\end{array}\right] \text { Cartesian } \\
=\left[\begin{array}{ccc}
\partial v_{r} / \partial r & \partial v_{\theta} / \partial r & \partial v_{z} / \partial r \\
r^{-1}\left(\partial v_{r} / \partial \theta-v_{\theta}\right) & r^{-1}\left(\partial v_{\theta} / \partial \theta+v_{r}\right) & r^{-1}\left(\partial v_{z} / \partial \theta\right) \\
\partial v_{r} / \partial z & \partial v_{\theta} / \partial z & \partial v_{z} / \partial z
\end{array}\right] \text { Cylindrical } \\
=\left[\begin{array}{ccc}
\partial v_{r} / \partial r & \partial v_{\theta} / \partial r & \partial v_{\phi} / \partial r \\
r^{-1}\left(\partial v_{r} / \partial \theta-v_{\theta}\right) & \left.r^{-1}\left(\partial v_{\theta} / \partial \theta\right)+v_{r}\right) & r^{-1}\left(\partial v_{\phi} / \partial \theta\right) \\
\frac{1}{r \sin \theta} \frac{\partial v_{r}}{\partial \phi}-\frac{v_{\phi}}{r} & \frac{1}{r \sin \theta} \frac{\partial v_{\theta}}{\partial \phi}-\frac{v_{\phi} \cot \theta}{r} & \left(\frac{1}{r \sin \theta} \frac{\partial v_{\phi}}{\partial \phi}+\frac{v_{r}}{r}+\frac{v_{\theta} \cot \theta}{r}\right)
\end{array}\right] \quad \text { Spherical. }
\end{gathered}
$$


We also need the divergence of a tensor of rank two. For a symmetrical tensor $\mathcal{T}$,

$$
\begin{gathered}
\nabla \cdot \mathcal{T}=\left(\frac{\partial \tau_{x x}}{\partial x}+\frac{\partial \tau_{x y}}{\partial y}+\frac{\partial \tau_{x z}}{\partial z}\right) \hat{x}+\left(\frac{\partial \tau_{x y}}{\partial x}+\frac{\partial \tau_{y y}}{\partial y}+\frac{\partial \tau_{y z}}{\partial z}\right) \hat{y}+\left(\frac{\partial \tau_{x z}}{\partial x}+\frac{\partial \tau_{y z}}{\partial y}+\frac{\partial \tau_{z z}}{\partial z}\right) \hat{z} \text { Cartesian } \\
=\left[\frac{1}{r} \frac{\partial\left(r \tau_{r r}\right)}{\partial r}+\frac{1}{r} \frac{\partial \tau_{r \theta}}{\partial \theta}-\frac{\tau_{\theta \theta}}{r}+\frac{\partial \tau_{r z}}{\partial z}\right] \hat{r}+\left[\frac{1}{r} \frac{\partial \tau_{\theta \theta}}{\partial \theta}+\frac{\partial \tau_{r \theta}}{\partial r}+\frac{2 \tau_{r \theta}}{r}+\frac{\partial \tau_{\theta z}}{\partial z}\right] \hat{\theta} \\
+\left[\frac{1}{r} \frac{\partial\left(r \tau_{r z}\right)}{\partial r}+\frac{1}{r} \frac{\partial \tau_{\theta z}}{\partial \theta}+\frac{\partial \tau_{z z}}{\partial z}\right] \hat{z} \quad \text { Cylindrical } \\
=\left[\frac{1}{r^{2}} \frac{\partial\left(r^{2} \tau_{r r}\right)}{\partial r}+\frac{1}{r \sin \theta} \frac{\partial\left(\tau_{r \theta} \sin \theta\right)}{\partial \theta}+\frac{1}{r \sin \theta} \frac{\partial \tau_{r \phi}}{\partial \phi}-\frac{\tau_{\theta \theta}+\tau_{\phi \phi}}{r}\right] \hat{r} \\
+\left[\frac{1}{r^{2}} \frac{\partial\left(r^{2} \tau_{r \theta}\right)}{\partial r}+\frac{1}{r \sin \theta} \frac{\partial\left(\tau_{\theta \theta} \sin \theta\right)}{\partial \theta}+\frac{1}{r \sin \theta} \frac{\partial \tau_{\theta \phi}}{\partial \phi}+\frac{\tau_{r \theta}}{r}-\frac{\tau_{\phi \phi} \cot \theta}{r}\right] \hat{\theta} \\
+\left[\frac{1}{r^{2}} \frac{\partial\left(r^{2} \tau_{r \phi}\right)}{\partial r}+\frac{1}{r} \frac{\partial \tau_{\theta \phi}}{\partial \theta}+\frac{1}{r \sin \theta} \frac{\partial \tau_{\phi \phi}}{\partial \phi}+\frac{\tau_{r \phi}}{r}+\frac{2 \tau_{\theta \phi} \cot \theta}{r}\right] \hat{\phi} \quad \text { Spherical. } \quad(A-7)
\end{gathered}
$$

There are several vector and tensor identities that are useful in fluid dynamics.

$$
\begin{gathered}
\mathcal{T}: \nabla \mathbf{v}=\nabla \cdot(\mathcal{T} \cdot \mathbf{v})-\mathbf{v} \cdot(\nabla \cdot \mathcal{T}) \\
\nabla \cdot(\rho \mathbf{u v})=(\nabla \rho \cdot \mathbf{u}) \mathbf{v}+\rho(\nabla \cdot \mathbf{u}) \mathbf{v}+\rho(\mathbf{u} \cdot \nabla) \mathbf{v} \\
(\mathbf{u} \cdot \nabla) \mathbf{u}=\frac{1}{2} \nabla(\mathbf{u} \cdot \mathbf{u})-\mathbf{u} \times(\nabla \times \mathbf{u}) \\
\mathbf{u} \cdot[\mathbf{u} \times(\nabla \times \mathbf{u})]=0
\end{gathered}
$$

There are several more vector identities of general interest.

$$
\begin{gathered}
\mathbf{a} \cdot(\mathbf{b} \times \mathbf{c})=\mathbf{b} \cdot(\mathbf{c} \times \mathbf{a})=\mathbf{c} \cdot(\mathbf{a} \times \mathbf{b}) \\
\mathbf{a} \times(\mathbf{b} \times \mathbf{c})=(\mathbf{a} \cdot \mathbf{c}) \mathbf{b}-(\mathbf{a} \cdot \mathbf{b}) \mathbf{c} \\
(\mathbf{a} \times \mathbf{b}) \cdot(\mathbf{c} \times \mathbf{d})=(\mathbf{a} \cdot \mathbf{c})(\mathbf{b} \cdot \mathbf{d})-(\mathbf{a} \cdot \mathbf{d})(\mathbf{b} \cdot \mathbf{c}) \\
\nabla \times \nabla \psi=0 \\
\nabla \cdot(\nabla \times \mathbf{a})=0 \\
\nabla \times(\nabla \times \mathbf{a})=\nabla(\nabla \cdot \mathbf{a})-\nabla^{2} \mathbf{a} \\
\nabla \cdot(\psi \mathbf{a})=\mathbf{a} \cdot \nabla \psi+\psi \nabla \cdot \mathbf{a} \\
\nabla \times(\psi \mathbf{a})=\nabla \psi \times \mathbf{a}+\psi \nabla \times \mathbf{a} \\
\nabla(\mathbf{a} \cdot \mathbf{b})=(\mathbf{a} \cdot \nabla) \mathbf{b}+(\mathbf{b} \cdot \nabla) \mathbf{a}+\mathbf{a} \times(\nabla \times \mathbf{b})+\mathbf{b} \times(\nabla \times \mathbf{a}) \\
\nabla \cdot(\mathbf{a} \times \mathbf{b})=\mathbf{b} \cdot(\nabla \times \mathbf{a})-\mathbf{a} \cdot(\nabla \times \mathbf{b})
\end{gathered}
$$




$$
\begin{gathered}
\nabla \times(\mathbf{a} \times \mathbf{b})=\mathbf{a}(\nabla \cdot \mathbf{b})-\mathbf{b}(\nabla \cdot \mathbf{a})+(\mathbf{b} \cdot \nabla) \mathbf{a}-(\mathbf{a} \cdot \nabla) \mathbf{b} \\
\mathbf{a} \cdot(\mathbf{b} \cdot \nabla) \mathbf{c}=\mathbf{a b}: \nabla \mathbf{c}
\end{gathered}
$$

Let $\mathbf{x}$ be a position vector with magnitude $r=|\mathbf{x}|$, and let $\mathbf{n}=\mathbf{x} / r$ be a unit radial vector. Then

$$
\begin{gathered}
\nabla \cdot \mathbf{x}=3 \\
\nabla \times \mathbf{x}=0 \\
\nabla \cdot \mathbf{n}=2 / r \\
\nabla \times \mathbf{n}=0 \\
(\mathbf{a} \cdot \nabla) \mathbf{n}=\frac{1}{r}[\mathbf{a}-\mathbf{n}(\mathbf{a} \cdot \mathbf{n})] \equiv \frac{\mathbf{a}_{\perp}}{r}
\end{gathered}
$$

There are also several integral theorems of interest. In the following, $\phi, \psi$, and $\mathbf{A}$ are well-behaved scalar or vector functions. $V$ is a three-dimensional volume bounded by a closed surface $S$ with area element $d a$ and outward unit normal $\mathbf{n}$. The volume elements of $V$ are $d^{3} x$. Then the following theorems hold.

$$
\begin{gathered}
\int_{V} \nabla \cdot \mathbf{A} d^{3} x=\oint_{S} \mathbf{A} \cdot \mathbf{n} d a \quad \text { (Divergence theorem) } \\
\int_{V} \nabla \psi d^{3} x=\oint_{S} \psi \mathbf{n} d a \\
\int_{V} \nabla \times \mathbf{A} d^{3} x=\oint_{S} \mathbf{n} \times \mathbf{A} d a \\
\int_{V}\left(\phi \nabla^{2} \psi+\nabla \phi \cdot \nabla \psi\right) d^{3} x=\oint_{S} \phi \mathbf{n} \cdot \nabla \psi d a \quad \text { (Green's first identity) } \\
\int_{V}\left(\phi \nabla^{2} \psi-\psi \nabla^{2} \phi\right) d^{3} x=\oint_{S}(\phi \nabla \psi-\psi \nabla \phi) \cdot \mathbf{n} d a \quad \text { (Green's theorem) }
\end{gathered}
$$

For $\mathcal{T}$, a tensor of rank two, the divergence theorem is

$$
\iiint \nabla \cdot \mathcal{T} d^{3} \mathbf{r}^{\prime}=\iint \hat{n} \cdot \mathcal{T} d^{2} a
$$

For the next two theorems, $S$ is an open surface with unit normal $\mathbf{n}$ defined by the right-hand side rule relative to the sense of the line integral around $C$, the contour bounding $S$. $C$ has the line element $d \mathbf{l}$.

$$
\begin{gathered}
\int_{S}(\nabla \times \mathbf{A}) \cdot \mathbf{n} d a=\oint_{C} \mathbf{A} \cdot d \mathbf{l} \quad\left(\text { Stokes }^{\prime} \text { theorem }\right) \\
\int_{S} \mathbf{n} \times \nabla \psi d a=\oint_{C} \psi d \mathbf{l}
\end{gathered}
$$

Finally, we have Leibnitz' rule.

$$
\frac{d}{d t} \int_{a(t)}^{b(t)} f(x, t) d x=\int_{a(t)}^{b(t)} \frac{\partial f(x, t)}{\partial t} d x+f(b(t), t) \frac{d b(t)}{d t}-f(a(t), t) \frac{d a(t)}{d t} .
$$




\section{B Commutation of LES Averaging and Differentiation}

Let us assume we have given a filter function $G\left(\mathbf{r}, \mathbf{r}^{\prime} ; L\right)$ which is an even function of $\mathbf{r}^{\prime}-\mathbf{r}$. Let us assume the smoothing leng $L$ is a constant. Our average density is

$$
\tilde{\rho}(\mathbf{r}, t) \equiv\langle\rho\rangle=\int_{-\infty}^{+\infty} \int_{-\infty}^{+\infty} \int_{-\infty}^{+\infty} \rho\left(\mathbf{r}^{\prime}, t\right) G\left(\mathbf{r}^{\prime}-\mathbf{r} ; L\right) d^{3} \mathbf{r}^{\prime}, \quad(B-1)
$$

and the averaged pressure is

$$
\tilde{P}(\mathbf{r}, t) \equiv\langle P\rangle=\int_{-\infty}^{+\infty} \int_{-\infty}^{+\infty} \int_{-\infty}^{+\infty} P\left(\mathbf{r}^{\prime}, t\right) G\left(\mathbf{r}^{\prime}-\mathbf{r} ; L\right) d^{3} \mathbf{r}^{\prime} . \quad(B-2)
$$

The mass-weighted average velocity is defined by

$$
\tilde{\rho}(\mathbf{r}, t) \overline{\mathbf{u}} \equiv\langle\rho \mathbf{u}\rangle=\int_{-\infty}^{+\infty} \int_{-\infty}^{+\infty} \int_{-\infty}^{+\infty} \rho\left(\mathbf{r}^{\prime}, t\right) \mathbf{u}\left(\mathbf{r}^{\prime}, t\right) G\left(\mathbf{r}^{\prime}-\mathbf{r} ; L\right) d^{3} \mathbf{r}^{\prime}
$$

Since the averaging operator is independent of time, it is obvious by Leibnitz' rule that it commutes with partial differentiation with respect to time:

$$
\left\langle\frac{\partial \rho}{\partial t}\right\rangle=\frac{\partial}{\partial t}\langle\rho\rangle=\frac{\partial \tilde{\rho}}{\partial t} .
$$

Commutation with the gradient and divergence operators is only slightly more difficult to prove. Consider first the pressure gradient. Note first that

$$
\nabla G\left(\mathbf{r}^{\prime}-\mathbf{r} ; L\right)=-\nabla^{\prime} G\left(\mathbf{r}^{\prime}-\mathbf{r} ; L\right)
$$

where $\nabla^{\prime}$ is the gradient with respect to the primed coordinates and $\nabla$ is the gradient with respect to the unprimed coordinates. Then

$$
\begin{gathered}
\left\langle\nabla^{\prime} P\left(\mathbf{r}^{\prime}, t\right)\right\rangle=\iiint \nabla^{\prime} P G\left(\mathbf{r}^{\prime}-\mathbf{r} ; L\right) d^{3} \mathbf{r}^{\prime} \\
=\iiint\left\{\nabla^{\prime}\left[P\left(\mathbf{r}^{\prime}, t\right) G\left(\mathbf{r}^{\prime}-\mathbf{r} ; L\right)\right]-P\left(\mathbf{r}^{\prime}, t\right) \nabla^{\prime} G\left(\mathbf{r}^{\prime}-\mathbf{r} ; L\right)\right\} d^{3} \mathbf{r}^{\prime} \\
=\iint_{S} P\left(\mathbf{r}^{\prime}, t\right) G\left(\mathbf{r}^{\prime}-\mathbf{r} ; L\right) \mathbf{n} d^{2} s^{\prime}+\iiint P\left(\mathbf{r}^{\prime}, t\right) \nabla G\left(\mathbf{r}^{\prime}-\mathbf{r} ; L\right) d^{3} \mathbf{r}^{\prime} \\
=\nabla \iiint P\left(\mathbf{r}^{\prime}, t\right) G\left(\mathbf{r}^{\prime}-\mathbf{r} ; L\right) d^{3} \mathbf{r}^{\prime}=\nabla \tilde{P}(\mathbf{r}, t) .
\end{gathered}
$$

The surface integral vanishes by the vanishing of $G$ at large distances, and $\nabla$ commutes with the integration by Leibnitz' rule.

It is a simple exercise to show that the averaging operator commutes with the divergence operator applied to either a vector or a rank two tensor. The proof follows the same line of reasoning as for the gradient, but with slightly different chain rules for differentiating a product. For a dyadic ab the proof is

$$
\left\langle\nabla^{\prime} \cdot\left[\mathbf{a}\left(\mathbf{r}^{\prime}, t\right) \mathbf{b}\left(\mathbf{r}^{\prime}, t\right)\right]\right\rangle=\iiint \nabla^{\prime} \cdot\left[\mathbf{a}\left(\mathbf{r}^{\prime}, t\right) \mathbf{b}\left(\mathbf{r}^{\prime}, t\right)\right] G\left(\mathbf{r}^{\prime}-\mathbf{r} ; L\right) d^{3} \mathbf{r}^{\prime}
$$




$$
\begin{aligned}
= & \iiint \nabla^{\prime} \cdot\left[\mathbf{a}\left(\mathbf{r}^{\prime}, t\right) \mathbf{b}\left(\mathbf{r}^{\prime}, t\right) G\left(\mathbf{r}^{\prime}-\mathbf{r} ; L\right)\right] d^{3} \mathbf{r}^{\prime}-\iiint \nabla^{\prime} G\left(\mathbf{r}^{\prime}-\mathbf{r} ; L\right) \cdot\left[\mathbf{a}\left(\mathbf{r}^{\prime}, t\right) \mathbf{b}\left(\mathbf{r}^{\prime}, t\right)\right] d^{3} \mathbf{r}^{\prime} \\
= & \iint \mathbf{n} \cdot\left[\mathbf{a}\left(\mathbf{r}^{\prime}, t\right) \mathbf{b}\left(\mathbf{r}^{\prime}, t\right)\right] G\left(\mathbf{r}^{\prime}-\mathbf{r} ; L\right) d^{2} s+\iiint \nabla G\left(\mathbf{r}^{\prime}-\mathbf{r} ; L\right) \cdot\left[\mathbf{a}\left(\mathbf{r}^{\prime}, t\right) \mathbf{b}\left(\mathbf{r}^{\prime}, t\right)\right] d^{3} \mathbf{r}^{\prime} \\
& =0+\nabla \cdot \iiint \mathbf{a}\left(\mathbf{r}^{\prime}, t\right) \mathbf{b}\left(\mathbf{r}^{\prime}, t\right) G\left(\mathbf{r}^{\prime}-\mathbf{r} ; L\right) d^{3} \mathbf{r}^{\prime}=\nabla \cdot\langle\mathbf{a}(\mathbf{r}, t) \mathbf{b}(\mathbf{r}, t)\rangle .
\end{aligned}
$$

To prove commutation for the divergence of a vector, simply replace $\mathbf{b}$ in Eq. (B-7) by the scalar 1.

The proof that the averaging operator commutes with the gradient and divergence operators requires that the surface integral of the filter function times some other function vanish. For infinite domains and periodic boundaries, cases for which the fluid variables are defined on all space, this condition is satisfied easily by the vanishing of the filter function at infinity (if not at a finite distance from $\mathbf{r}$ ). The situation is not so clear for confined flows, especially with no-slip boundaries, where filters without compact support will cross every boundary for all points in the computational domain. Even with compact support, the filter function will intersect a boundary for points sufficiently close to the boundary. This is a weakness of the filter function approach that we shall not consider further in this report.

Another weakness of the filter function approach appears when we consider filters in which $L$ is a function of position or time. Then the filter function $G\left(\mathbf{r}-\mathbf{r}^{\prime}, L(\mathbf{r}, t)\right)$ depends on time and on $\mathbf{r}$ in a way that is not symmetric with $\mathbf{r}^{\prime}$. This situation will occur on all non-uniform grids if $L$ is tied to the local grid size, especially in non-Eulerian calculations. In these cases, equations (B-4) through (B-7) are not valid. For example, consider the time derivative of the density when $L$ is a function of time:

$$
\left\langle\frac{\partial \rho}{\partial t}\right\rangle=\frac{\partial \tilde{\rho}}{\partial t}-\int_{-\infty}^{\infty} \rho\left(\mathbf{x}^{\prime}, t\right) \frac{\partial G\left(\mathbf{x}-\mathbf{x}^{\prime}, L(\mathbf{x}, t)\right)}{\partial t} d^{3} x^{\prime}
$$

One can formally write down all of the missing terms, but these are a new set of turbulence functionals involving derivatives of $G$, and it is not clear how to model them. Strictly speaking, our averaged equations are correct only for $L$ being a constant, even if we ignore the boundary problem discussed in the previous paragraph. In practice, it is a good idea not to let the mesh change more that a few percent from one zone to the next. Experience suggests that this modest rate of change does not appear to cause problems at the 5 to 10 percent accuracy level. However, the more drastic grid variations of Lagrangian or Arbitrary Lagrangian-Eulerian (ALE) calculations may not be so benign. This issue is discussed in more detail by Ghosal and Moin [26]. 


\section{Nuclear Reaction Networks}

The chemical reactions occurring in the system are symbolized by

$$
\sum_{\alpha} a_{\alpha r} \chi_{\alpha} \rightleftharpoons \sum_{\alpha} b_{\alpha r} \chi_{\alpha}
$$

where $\chi_{\alpha}$ represents one mole of species $\alpha$, and $a_{\alpha r}$ and $b_{\alpha r}$ are the dimensionless stoichiometric coefficients for the $r$ th reaction. It is assumed that $a_{\alpha r}$ and $b_{\alpha r}$ are integers. The chemical source term in the species continuity equation is given by

$$
R_{\alpha}=W_{\alpha} \sum_{r}\left(b_{\alpha r}-a_{\alpha r}\right) \dot{\omega}_{r}
$$

where $\dot{\omega}_{r}$ is the rate of progress of the $r$ th reaction,

$$
\dot{\omega}_{r}=k_{f r} \prod_{\alpha}\left(\rho_{\alpha} / W_{\alpha}\right)^{a_{\alpha r}^{\prime}}-k_{b r} \prod_{\alpha}\left(\rho_{\alpha} / W_{\alpha}\right)^{b_{\alpha r}^{\prime}} .
$$

Here $k_{f r}$ and $k_{b r}$ are the forward and backward rate coefficients for reaction $r$, and the exponents $a_{\alpha r}^{\prime}$ and $b_{\alpha r}^{\prime}$ specify the order of the reaction. For an elementary reaction, $a_{\alpha r}^{\prime}=$ $a_{\alpha r}$ and $b_{\alpha r}^{\prime}=b_{\alpha r}$. The coefficients $k_{f r}$ and $k_{b r}$ are assumed to be of a generalized Arrhenius form:

$$
\begin{aligned}
k_{f r} & =A_{f r} T^{\zeta_{f r}} \exp \left(-E_{f r} / T\right), & & (C-4) \\
k_{b r} & =A_{b r} T^{\zeta_{b r}} \exp \left(-E_{b r} / T\right), & & (C-5)
\end{aligned}
$$

where $E_{f r}$ and $E_{b r}$ are activation temperatures. It is clear that functionals such as $\left\langle R_{\alpha}\right\rangle$ are going to be difficult to model accurately and reliably.

\section{References}

[1] T. Cebeci and A. M. O. Smith, Analysis of Turbulent Boundary Layers (Academic Press, New York, 1974).

[2] M. Germano, "Turbulence: The filtering approach," J. Fluid Mech. 238, 325 (1992).

[3] W. C. Reynolds, "Computation of turbulent flows," Ann. Rev. Fluid Mech. 8, 183 (1976).

[4] W. C. Reynolds, "Modeling of fluid motions in engines - an introductory overview," in Combustion Modeling in Reciprocating Engines, ed. J. N. Mattavi and C. A. Amann (Plenum Press, New York, 1980), p. 41.

[5] B. E. Launder and D. B. Spalding, Mathematical Models of Turbulence (Academic Press, London, 1972). 
[6] B. E. Launder and D. B. Spalding, "The numerical computation of turbulent flows," Comp. Meth. App. Mech. Eng. 3, 269 (1974).

[7] W. P. Jones, "Models for turbulent flows with variable density and combustion," in Prediction Methods for Turbulent Flows, ed. W. Kollmann (Hemisphere, Washington, 1980), p. 379.

[8] W. P. Jones and B. E. Launder, "The prediction of laminarization with a two-equation model of turbulence," Int. J. Heat Mass Transfer 15, 301 (1972).

[9] U. Schumann, "Subgrid scale model for finite difference simulations of turbulent flows in plane channels and annuli," J. Comput. Phys. 18, 376 (1975).

[10] A. Favre, "Équations des gaz turbulents compressibles," J. de Mécanique 4, 361 (1965).

[11] A. Favre, "Statistical equations of turbulent gases," in Problems of Hydrodynamics and Continuum Mechanics (Society for Industrial and Applied Mathematics, Philadelphia, PA, 1969), p. 231.

[12] T. G. Cowling, "The stability of gaseous stars," Mon. Not. Royal Ast. Soc. 96, 42 (1935).

[13] L. H. Aller and S. Chapman, "Diffusion in the sun," Ap. J. 132, 461 (1960).

[14] R. B. Bird, W. E. Stewart, and E. N. Lightfoot, Transport Phenomena (Wiley, New York, 1960).

[15] S. Chapman and T. G. Cowling, The Mathematical Theory of Non-Uniform Gases (Cambridge University Press, London, 1953).

[16] J. D. Ramshaw, "Self-consistent effective binary diffusion in multicomponent gas mixtures," J. Non-Equilib. Thermodyn. 15, 295 (1990).

[17] J. D. Ramshaw and C. H. Chang, "Ambipolar Diffusion in Multicomponent Plasmas," Plasma Chem. Plasma Proc. 11, 395 (1991).

[18] M. W. Chase, Jr., C. A. Davies, J. R. Downey, Jr., D. J. Frurip, M. A. McDonald, and A. N. Syverud, "JANAF Thermochemical Tables, Third Edition, Parts I and II, Supplement No. 1," J. Phys. Chem. Ref. Data 14 (1985).

[19] J.-L. Tassoul, Theory of Rotating Stars (Princeton U. Press, Princeton, NJ, 1978). 
[20] S. Chandrasekhar, An Introduction to the Study of Stellar Structure (University of Chicago Press, Chicago, 1939).

[21] R. G. Deupree, "Another fundamental, but correctable, inconsistency in the local mixing-length theory," Ap. J. 234, 228 (1979).

[22] L. D. Cloutman, "Ensemble-averaged thermonuclear reaction rates for turbulent convection in stellar interiors," Ap. J. 437, 384 (1994).

[23] K. Horiuti, "A new dynamic two-parameter mixed model for large-eddy simulation," Phys. Fluids 9, 3443 (1997).

[24] J. Smagorinsky, "General circulation experiments with the primitive equations: I. The basic experiment," Mon. Weather Rev. 91, 99 (1963).

[25] P. H. Gaskell and K. H. C. Lau, "Pressure-containing correlations in variable density and turbulent reacting flows," Comb. \& Flame 73, 215 (1988).

[26] S. Ghosal and P. Moin, "The basic equations for the large eddy simulation of turbulent flows in complex geometry," J. Comput. Phys. 118, 24 (1995). 